\title{
TESTUDINOIDEA FITZINGER (TESTUDINES: CRYPTODIRA) DE LA FORMACIÓN TOROPÍ/YUPOÍ (CA. 58-28 KA) EN LA PROVINCIA DE CORRIENTES, ARGENTINA: TAXONOMÍA Y ASPECTOS PALEOAMBIENTALES
}

\author{
GERARDO GABRIEL ZACARÍAS \\ CONICET, Facultad de Ciencias Naturales, Instituto de Biogeociencias del Noroeste, Universidad Nacional de Salta,
} Salta, Argentina.gzpaleozacarias@gmail.com

MARCELO SAÚL DE LA FUENTE

CONICET, Departamento de Paleontología Museo de Historia Natural de San Rafael, Parque Mariano Moreno s/n, San Rafael, Mendoza, Argentina.mdelafuente1910@gmail.com

ALFREDO EDUARDO ZURITA

CONICET, Centro de Ecología Aplicada del Litoral y Universidad Nacional del Nordeste, Ruta 5 km 2,5 3400, Corrientes, Argentina.aezurita@yahoo.com.ar

\begin{abstract}
TESTUDINOIDEA FITZINGER (TESTUDINES: CRYPTODIRA) OF THE TOROPÍ/YUPOÍ FORMATION (CA. 58-28 KA) IN THE PROVINCE OF CORRIENTES, ARGENTINA: TAXONOMY AND PALEOENVIRONMENTAL ASPECTS. Remains of testudinoids from Toropí/Yupoí Formation ( $c a .58-28 \mathrm{ka}$ ), including testudinids and emydids, were recovered from Arroyo Toropí and Santa Lucía localities (Corrientes Province, northeastern Argentina). From Arroyo Toropí two specimens of testudinid are known: the holotype of Chelonoidis lutzae (carapace, plastron and remains of the appendicular skeleton) and a partial carapace tentatively referred to this species. New specimens including remains of testudinids (cf. Chelonoidis) and emydids (Trachemys cf. T. dorbigni) have been also recovered from Santa Lucía. Comparing the global distribution of extant species of giant testudinids (e.g. Chelonoidis nigra, Geochelone sulcata, Dipsochelys elephantina and Cylindraspis peltastes) with the climate map of Kottek and collaborators, show congruence with the Tropical and Subtropical zones, characterized by warm climate. This suggests a high degree of thermosensitivity for these extant giant tortoises. This, together with the geological and paleontological data from Toropí/Yupoí Formation, allows suggesting that the giant Chelonoidis inhabited environments with warm climate conditions. In addition, warm and wet conditions are inferred in Santa Lucía from the record of Trachemys cf. dorbigni.
\end{abstract}

Key words: Testudinoidea, Testudinidae, Emydidae, Late Pleistocene, Corrientes, Argentina.

RESUMO - Os testudinoideos recuperados da Formação Toropí/Yupoí (ca. 58-28 ka) estão representados por testudinídeos e emidídeos. Estes foram coletados em duas localidades (Província de Corrientes, Argentina): Arroyo Toropí e Santa Lucía. Da primeira localidade procedem dois testudinídeos: o holótipo de Chelonoidis lutzae, composto por partes da carapaça dorsal, plastrão e restos do esqueleto apendicular, e outro exemplar tentativamente assignado a esta espécie que está representado por fragmentos de carapaça dorsal e plastrão. Novos exemplares constituídos por restos fragmentários de testudinídeos (cf. Chelonoidis) e emidídeos (Trachemys cf. dorbigni) procedem de Santa Lucía. A partir da constatação de mapas de distribuição global de espécies atuais de testudinídeos gigantes (e.g. Chelonoidis nigra, Geochelone sulcata, Dipsochelys elephantina y Cylindraspis peltastes) e o mapa climatológico de Kottek e colaboradores se observa una congruência entre a distribuição geográfica destas espécies gigantes atuais com as zonas tropical e subtropical caracterizadas por climas quentes, indicando um alto grau de sensibilidade térmica para estas tartarugas terrestres. Esta informação, somada aos dados geológicos e paleontológicos da Formação Toropí/Yupoí, sugere que os Chelonoidis gigantes habitaram na área do Arroyo Toropí e Santa Lucía sob condições climáticas quentes. Por sua vez, condições quentes e úmidas são inferidas a partir da presença de Trachemys cf. T. dorbigni na mesma unidade aflorante em Santa Lucía.

Palavras-chave: Testudinoidea, Testudinidae, Emydidae, Pleistoceno final, Corrientes, Argentina. 


\section{INTRODUCCIÓN}

En el extremo meridional de América del Sur, los testudinoideos (Testudines: Cryptodira) están restringidos a Emydidae Bell, 1825 y Testudinidae Gray, 1825 (Joyce et al., 2004; Van Dijk et al. 2011). La familia Testudinidae es un clado de tortugas terrestres que está representado en América del Sur por el género Chelonoidis Fitzinger, 1835. Este género posee especies actuales y extintas. Entre las primeras es posible reconocer a: Ch. chilensis (Gray, 1870), Ch. petersi (Freiberg, 1973; Fernández, 1988; contra Fritz et al., 2012a), Ch. carbonaria (Spix, 1824), Ch. denticulata (Linnaeus, 1766) y Ch. nigra (Quoy \& Gaimard, 1824). Entre las especies extintas atribuidas a este género se reconocen algunas de pequeño a mediano tamaño (Ch. gringorum Simpson, 1942, Ch. hesterna Auffenberg, 1971) y otras especies gigantes (?Ch. gallardoi Rovereto, 1914, ?Ch. australis Moreno, 1889 y Ch. lutzae Zacarías, De la Fuente, Fernández \& Zurita, 2013) (Broin \& De la Fuente, 1993; De la Fuente, 1994; De la Fuente, 1997). A pesar que otras especies pleistocenas además de Ch. lutzae (por ejemplo "Testudo" sellowii Weis, 1830 o presuntamente pleistocénicas como "Testudo" elata Gervais, 1877) fueron nominadas en el siglo XIX, su naturaleza fragmentaria impide por el momento validar las mismas. Por otra parte, la familia Emydidae (un grupo de tortugas cryptodiras dulceacuícolas) está representada en América del Sur por dos especies vivientes: T. ornata y T. dorbigni (Fritz et al., 2012b). Esta última especie distribuida en el nordeste de Argentina, en Brasil y Uruguay (Richard, 2009; Van Dijk et al., 2011; Fritz et al., 2012b); presenta registro fósil conocido en el Pleistoceno de localidades del Nordeste (provincias de Formosa y Entre Ríos) y Centro (Provincia de Córdoba) de Argentina (De la Fuente, 1999; De la Fuente et al., 2002; Cabrera \& Luna, 2011).

En esta contribución, se dan a conocer registros de testudinoideos (representados por restos de Testudinidae y Emydidae) provenientes de dos localidades en la Provincia de Corrientes: Una localidad ya conocida Arroyo Toropí y otra nueva localidad fosilífera, Santa Lucía (Figura 1). Por lo tanto, estos hallazgos amplían el registro de esta superfamilia para el Pleistoceno tardío de América del Sur. De modo adicional, se analizará el potencial de los ejemplares de Testudinoideos como indicadores paleoclimáticos y paleoambientales vinculados a la Formación Toropí/Yupoí (sensu Herbst, 1971; Iriondo, 1973; 2010; Iriondo \& Krohling, 2008 y Tonni, 2009). De esta manera, se testeará cuál de las hipótesis propuestas sobre las condiciones paleoclimáticas y paleoambientales de la Formación Toropí/ Yupoí se ajustaría más la información obtenida a partir del estudio de estos ejemplares testudinoideos.

\section{CONTEXTO GEOLÓGICO, ESTRATIGRÁFICO Y PALEOAMBIENTAL}

Las localidades donde fueron extraídos los ejemplares de Testudinoidea aquí presentados son: Arroyo Toropí y Santa Lucía, en la Provincia de Corrientes, Argentina. En estas localidades aflora la Formación Toropí/ Yupoí. Esta unidad litológica, se distribuye sobre la margen Este del río Paraná, suprayaciendo a la Formación Ituzaingó (Mioceno tardío ca. 7-2 Ma - Presente sensu Iriondo, 2010). Inicialmente, Herbst (1971) nominó a esta unidad como Formación Yupoí, dividiéndola en dos miembros. Posteriormente, Herbst \& Álvarez (1974) utilizaron mamíferos fósiles para dividir esta unidad en dos formaciones, Toropí [e.g. Theriodictis sp. y Scelidodon sp. (Piso/ Edad: Ensenadense sensu Pascual et al., 1965)] y Yupoí [Megatherium sp., Euphractus sp. y Equus sp. (Piso/ Edad: Lujanense sensu Pascual et al., 1965)]. Sin embargo, tal división mediante los biocrones de mamíferos no es posible ya que estudios posteriores amplían el biocrón de dichos mamíferos (Cione \& Tonni, 2005; Carlini et al., 2008; Tonni 2009; Alcaraz, 2010; Francia et al., 2012a,b) que utilizaron Herbst \& Alvarez (1974). Por otro lado, Iriondo (1973, 2010) e Iriondo \& Krohling (2008) mediante análisis mineralógico postularon que ambas unidades formacionales corresponderían a una sola (en congruencia con Herbst, 1971), nominándola Formación Toropí/ Yupoí.

La Formación Toropí/ Yupoí está compuesta por arenas arcillosas, limos arenosos y arcillas arenosas en proporciones variables (Herbst, 1971). Aquí se reconocen dos miembros; el inferior posee arenas duras de colores gris amarillento a verdoso, dispuestas en bancos a veces estratificados. Este está separado del miembro superior por una superficie de arena muy endurecida, denominada "costra silícea" o "duricostra" (sensu Scillato Yané et al., 1998). A su vez, el miembro superior se caracteriza por contener sedimentitas menos consolidadas, con mayor variedad litológica y de colores, desde rosado a rojo llegando a diferenciarse en algunas zonas dos secciones, donde la superior es más oscura. En Arroyo Toropí, Herbst (1971) utiliza la "costra silícea" para marcar el límite entre el miembro inferior del superior (Figura 2a), pero tal estructura sedimentaria no aparece en Santa Lucía (Iriondo, 1973) (Figura 2b).

Dataciones OSL (optically stimulated luminiscence) sugieren que la Formación Toropí/Yupoí se depositó durante el lapso 58-28 Ka, correlacionable con el MIS 3 (Oxigen marine isotope stages) (Tonni, 2009; Francia et al., 2012a). Es importante destacar que durante el MIS 3, el río Paraná tenía una posición diferente, ya que se encontraba en una posición más oriental (Iriondo \& Krohling, 2008; Iriondo, 2010; Orfeo \& Iriondo, 2010), posiblemente ocupando la extensión de los grandes paleocauces de los ríos Aguapey y Miriñay, en contacto con el río Uruguay (Iriondo \& Krohling, 2008; Iriondo, 2010; Orfeo \& Iriondo, 2010).

Desde una perspectiva paleoclimática y paleoambiental, han sido propuestas diferentes interpretaciones para comprender las condiciones en que se depositó esta unidad litoestratigráfica. Estas se pueden sintetizar, según se consideren autores que interpretaron esta secuencia pleistocena como compuestas por dos distintas unidades (formaciones Toropí y Yupoí) o por dos miembros de una misma formación (Formación Toropí/Yupoí). Los primeros postularon tres diferentes hipótesis: (1a) ambiente frío árido para la Formación Toropí y para la Formación Yupoí, ambiente 

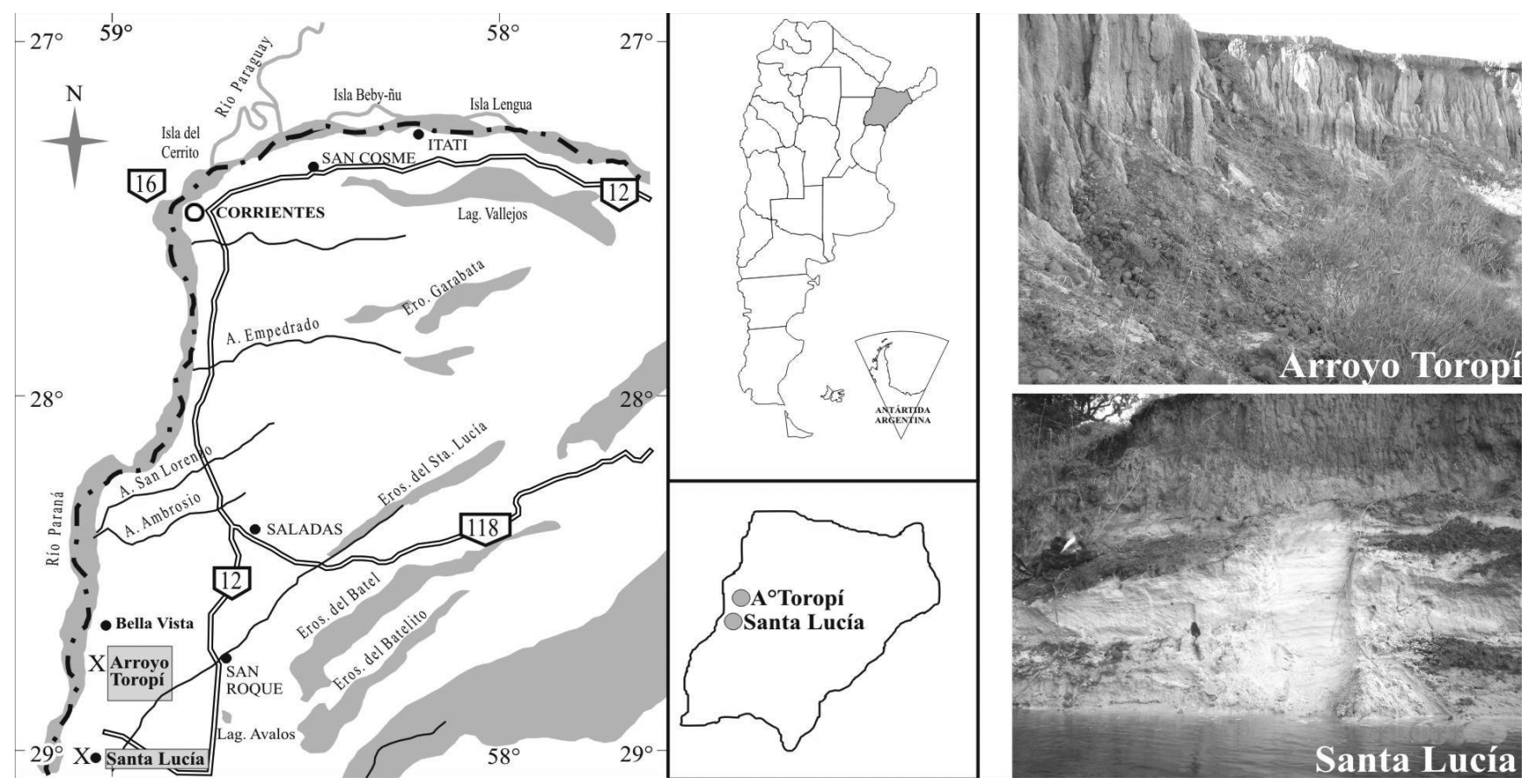

Figura 1. Mapa de Argentina donde se muestran las localidades fosilíferas de Arroyo Toropí y Santa Lucía (Provincia de Corrientes). Abreviaturas: $\mathbf{A}^{\circ}$, arroyo; Eros, esteros; Lag., lagunas.

Figure 1. Location map showing Arroyo Toropí and Santa Lucía localities (Corrientes Province). Abbreviations: $\mathbf{A}^{\circ}$, stream; Eros, marshes; Lag., lagoon.

A

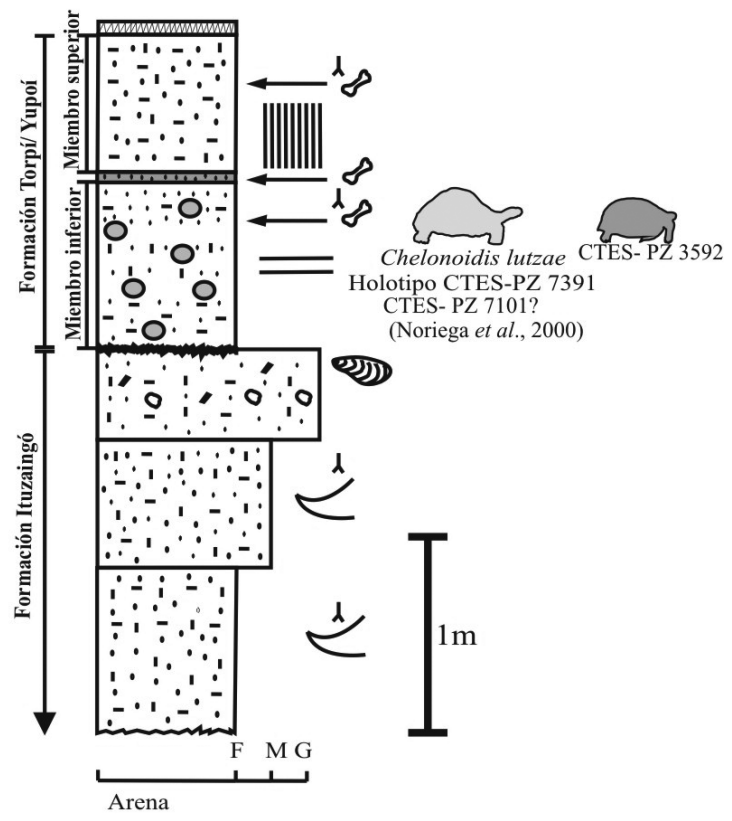

B

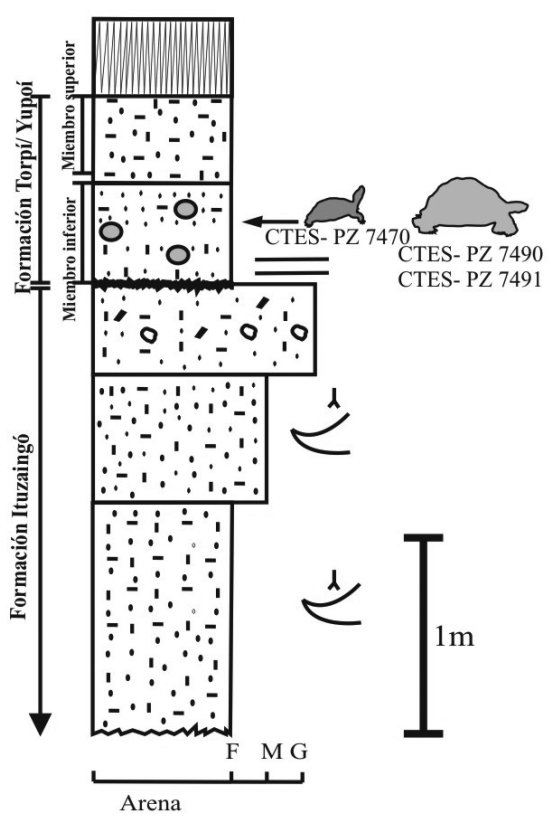

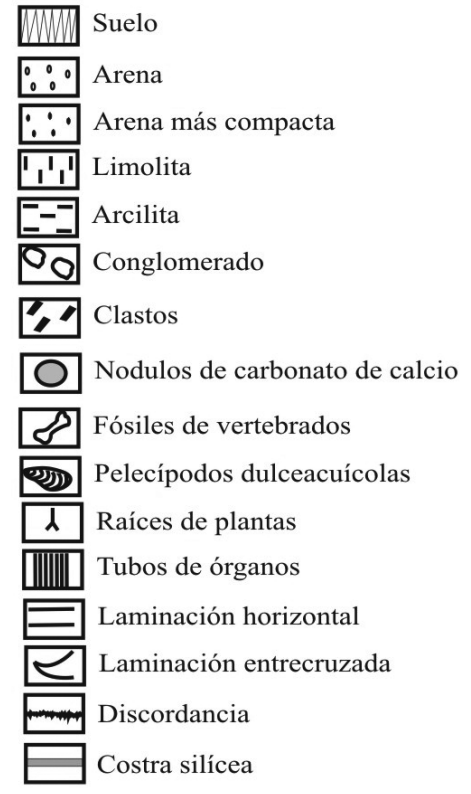

Figura 2. Perfiles estratigráficos elaborados a partir de las descripciones litológicas de Herbst (1971) e Iriondo (2010). A, Arroyo Toropí; B, Santa Lucía.

Figure 2. Stratigraphical profiles based on the contributions by Herbst (1971) and Iriondo (2010). A, Arroyo Toropí; B, Santa Lucía. 
más cálido y húmedo (Álvarez, 1974; Herbst \& Álvarez, 1974); (2a) distintas condiciones paleoclimáticas para la Formación Toropí con lapsos áridos/ fríos y otros más cálidos/ húmedos, en base a la interpretación de los requerimientos paleoambientales de la fauna de vertebrados registrada (Scillato Yané et al., 1998; Carlini et al., 2004; 2008; Francia et al., 2009; 2012b; Albino \& Carlini, 2008; Alcaraz, 2010); (3a) condiciones cálidas y húmedas con estacionalidad marcada, presentando leves a moderados niveles de saturación de la disponibilidad hídrica en suelos bien desarrollados con abundante vegetación para ambas formaciones, en base al análisis fitolítico de termiteros y sedimentitas (Erra et al., 2010; 2013). Por otra parte, autores que consideran una misma unidad litoestratigráfica (Formación Toropí/ Yupoí), han propuesto dos hipótesis: (1b) las condiciones en que se depositó la Formación Toropí/ Yupoí fueron cálidas y húmedas (Iriondo, 1973; 2010; Iriondo \& Krohling, 2008; Tonni, 2009); (2b) paleoambiente dinámico tipo ecotonal afectados por el cambio de las variables paleoclimáticas (Francia et al., 2012b).

Por otra parte, altas concentraciones de arcillas montmorillonitas e illitas respecto de las coallinitas encontradas en esta unidad (Herbst \& Santa Cruz, 1985), podrían evidenciar condiciones climáticas complejas con lapsos semiáridos con estaciones bien marcadas y lapsos de climas húmedos y templados. Iriondo (2007) postula que las montmorillonitas se forman en climas semiáridos con estaciones bien marcadas, en tanto que las illitas se generan en climas fríos o muy secos y las coallinitas en climas húmedos y templados. Esta evidencia mineralógica, es congruente con tres hipótesis paleoclimáticas y paleoambientales previas [hipótesis (3a); (1b); (2b)]. A su vez, se podría inferir condiciones ambientales cálidas y húmedas por la abundancia del helecho Equisetum sp. (orden Equisetales) en el miembro superior de la Formación Toropí/Yupoí (Lutz \& Gallego, 2001). Actualmente este género vive en suelos areno-arcilloso con altos niveles de saturación hídrica y sometidos a períodos de inundaciones (Husby, 2009). Por otro lado, Barreda et al. (2007) postularon que durante el Pleistoceno tardío en el actual territorio de la Provincia de Corrientes, perteneció a la gran región Neotropical más relacionado con la provincia fitogeográfica Chaqueña.

Consideramos que, posiblemente las condiciones climáticas complejas puedan explicarse en el contexto geoclimático del MIS 3 y del fenómeno del Niño y la Niña. Ya que Meerbeeck et al. (2009) postulan que las condiciones climáticas del MIS 3 respecto del Último Máximo Glacial [UMG, MIS 2] fueron $2^{\circ} \mathrm{C}$ más cálidas, registrándose alternancia de períodos fríos de $1,7^{\circ} \mathrm{C}$ MIS 3 -sta (estadiales) y cálidos de $2,0^{\circ} \mathrm{C}$ MIS3 (int interstadiales). Mientras que Martinez (2009) postula, que durante la transición del Pleistoceno medio- superior hubo un cambio entre las condiciones del Niño (clima más húmedo con mayores precipitaciones) a la Niña (clima más árido con bajas precipitaciones), mientras que durante el Último Máximo Glacial las condiciones fueron más complejas, predominando el clima tipo Niña con ocurrencias del fenómeno del Niño.

Abreviaturas institucionales. AMNH, American Museum of
Natural History, New York, USA; CTES-PZ, Paleozoología Corrientes, Facultad de Ciencias Exactas y Naturales y Agrimensura, Universidad Nacional del Nordeste, Correntes, Argentina; MACN, Museo Argentino de Ciencias Naturales "Bernardino Rivadavia", Buenos Aires, Argentina; MHNSR, Museo de Historia Natural San Rafael, Mendoza, Argentina; MLP, Museo de La Plata; MPEF, Museo Paleontológico "Egidio Feruglio", Chubut, Argentina; PC, Pritchard Collection, Chelonian Research Institute, Oviedo, Florida, USA; UNNEC, Colección de la Universidad Nacional del Nordeste, Corrientes, Argentina; YPFB- PAL, Yacimientos Petrolíferos Bolivianos-Paleontología, Bolivia.

Abreviaturas anatómicas. Para la terminología anatómica del caparazón se siguió a Zangerl (1969). Escudos: ab, abdominal; an, anal; f, femoral; g, gular; h, humeral; m, marginal; p, pectoral; pl, pleural; sc, supracaudal; $\mathbf{v}$, vertebral. Placas: C, costal; En, entoplastrón; EP, Epiplastrón; Ho, Hioplastrón; Hp, Hipoplastrón; N, neural; P, periférica; Pa, pilar axilar; Pi, pigal; xi, xifiplastrón.

\section{PALEONTOLOGÍA SISTEMÁTICA}

\author{
OrdenTESTUDINES Batsch, 1788 \\ Superfamilia TESTUDINOIDEA Fitzinger, 1826 \\ Familia TESTUDINIDAE Batsch, 1788
}

Chelonoidis Fitzinger, 1835

Especie tipo. Testudo carbonaria Spix, 1824.

Chelonoidis lutzae Zacarías, De la Fuente, Fernández \& Zurita, 2013

(Figuras 3A,B)

Holotipo. CTES-PZ 7391, caparazón dorsal y plastrón, escápula derecha e izquierda, coracoides derecho, ambos húmeros y fémures de un individuo subadulto (Figuras 3A,B).

Diagnosis. Ver Zacarías et al. (2013).

Medidas. Ver tabla 2 en Zacarías et al. (2013).

Descripción. Esta especie fue descrita en detalle por Zacarías et al. (2013).

Procedencia geográfica. El holotipo de esta especie provienen del Arroyo Toropí, Provincia de Corrientes, Argentina (2831'20,24”'S, 5903'34,91'O 58) (Figura 1).

Procedencia estratigráfica. Sección superior del miembro inferior de la Formación Toropí/Yupoí (Pleistoceno tardío, $c a$. 58-28ka) (Zacarías et al., 2013, figuras 1 y 2) (Figura 2A).

Comentarios. Zacarías et al. (2013) nominaron al ejemplar pleistoceno de tortuga terrestre gigante CTES-PZ 7391 como una nueva especie, Chelonoidis lutzae. A su vez estos autores, determinaron que el holotipo es un macho (Figuras 3A,B). Zacarías et al. (2013) plantean que otro ejemplar de tortuga terrestre gigante dado a conocer por Noriega et al. (2000) en la misma localidad y unidad litoestratigráfica, deja abierta la posibilidad de que ambos podrían corresponder al mismo taxón (Figura 3C). 
A

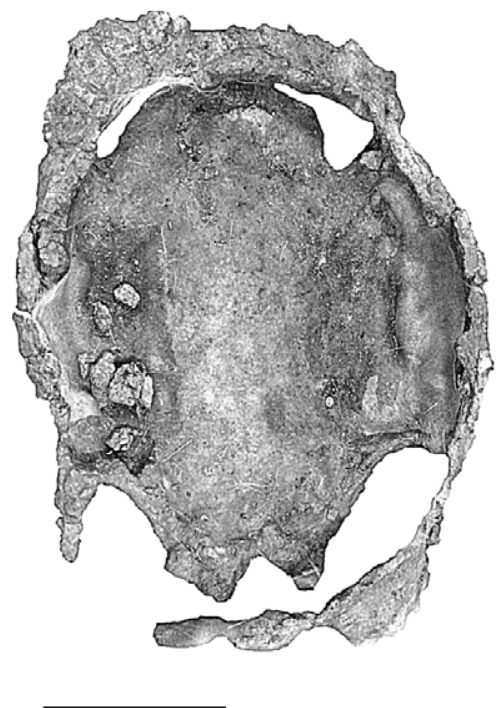

B

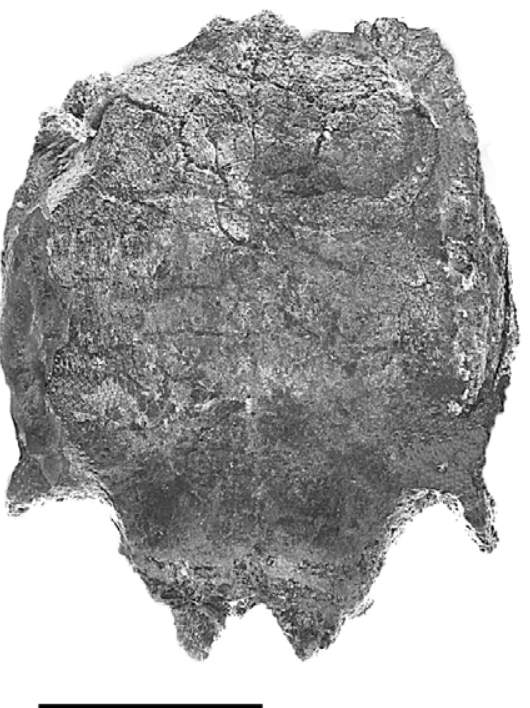

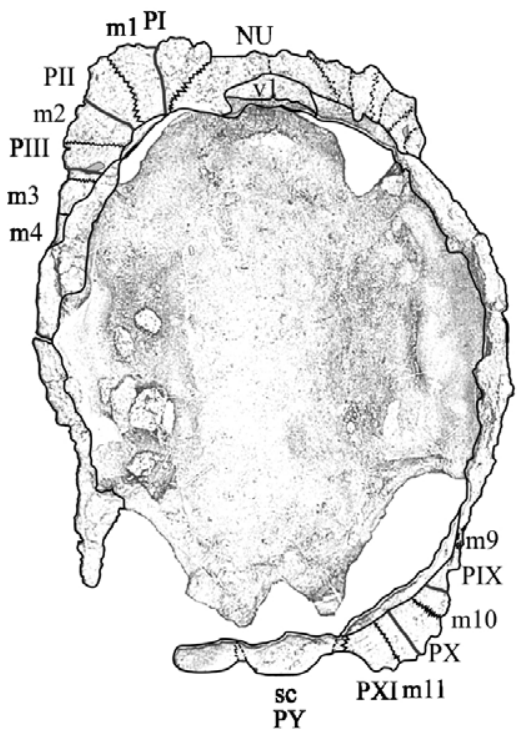

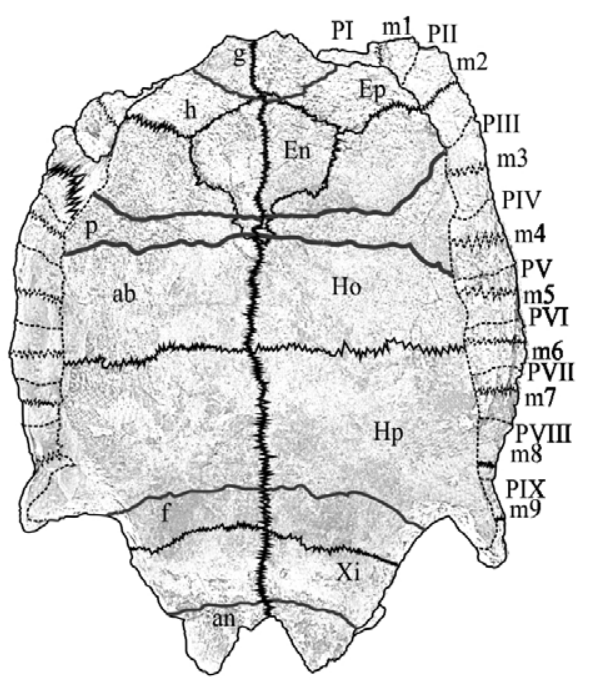

C
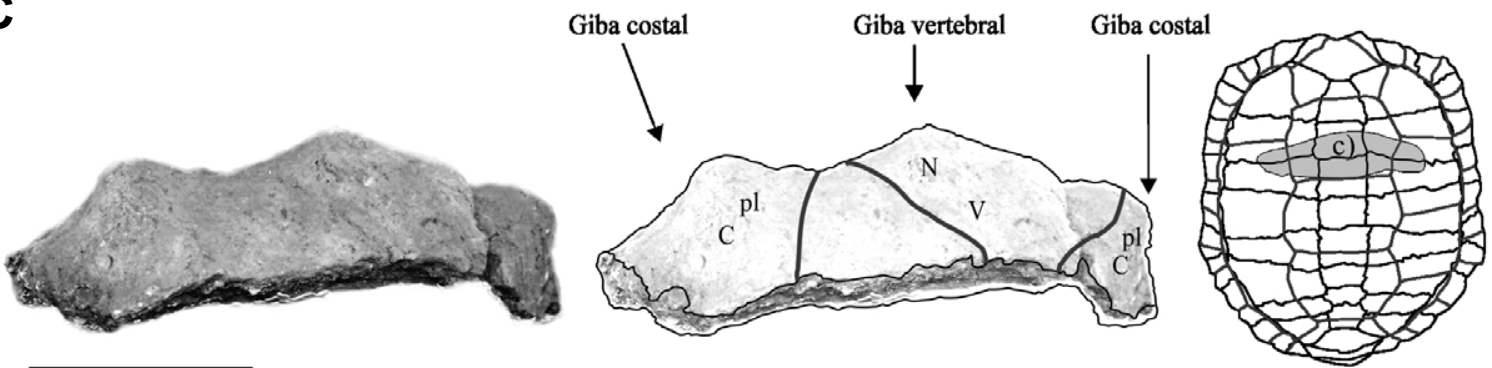

Figura 3. A-B, Holotipo de Chelonoidis lutzae (ver figuras 3 y 4 en Zacarías et al., 2013). A, vista dorsal del caparazón, izquierda, foto, derecha, esquema; B, vista ventral del plastrón, izquierda, foto, derecha esquema; C, vista anterior del caparazón del ejemplar CTES- PZ 7101, izquierda, foto; derecha, esquema; extremo derecho esquema de las partes preservadas (ver Noriega et al., 2000). Abreviaturas: Placas: C, costales; En, entoplastron; Ep, epiplastron; Ho, hioplastron; Hp, hipoplastrón; N, neurale; NU, nucal; $\mathbf{P}$, periférica; Py, pigal; $\mathbf{X i}$, xifiplastrón; Escudos: ab, abdominales; an, anales; $\mathbf{h}$, humerales; f, femorales; $\mathbf{g}$, gulares; $\mathbf{m}$, maginal; $\mathbf{p}$, pectorales; pl, pleural; sc, supracaudal; $\mathbf{v}$, vertebrales. Escalas: A-C $=150 \mathrm{~mm}$.

Figure 3. A-B, Chelonoidis lutzae holotype (see figures 3 and 4 in Zacarías et al., 2013). A, dorsal view of the caparace, picture, left, drawing, right; B, ventral view of the plastron, picture, left; drawing right; C, anterior view of the caparace of specimen CTES- PZ 7101, picture, left; scheme, right; scheme of preserved part, extreme right (see Noriega et al., 2000). Abbreviations: Plates: C, costals; En, entoplastron; Ep, epiplastron; Ho, hioplastron; Hp, hipoplastron; N, neural; NU, nuchal; P, periferic; Py, pygal; Xi, xifiplastron; Scutes: ab, abdominals; an, anals; h, humerals; f, femorals; g, gulars; m, maginal; p, pectorals; pl, pleural; sc, supracaudal; v, vertebral. Scale bars $=150 \mathrm{~mm}$. 
Chelonoidis lutzae representa el primer registro fósil de una especie válida de tortuga terrestre gigante para el Pleistoceno de América del Sur. Análisis filogenéticos realizados por Zacarías et al. (2013) establecen que Ch. lutzae junto con ?Ch. australis constituyen un clado claramente diferenciado de las tortugas gigantes de las islas Galápagos, al que denominaron informalmente como "tortugas terrestres gigantes continentales extintas".

\section{cf. Chelonoidis}

(Figuras 4,5)

Materiales referidos a cf. Chelonoidis. Ejemplares CTESPZ 3592; CTES- PZ 7491 y CTES-PZ 7490 (Figuras 4, 5).

Material de comparación. Apéndice 1.

Procedencia geográfica. Uno de los materiales (CTES- PZ 3592) provienen del Arroyo Toropí (Figuras 1, 2A); otros ejemplares (CTES- PZ 7491, CTES- PZ 7490) provienen de la localidad de Santa Lucía (2859'07, 32”'S, 5906'03, 89”O) (Figuras 1,2B).

Procedencia estratigráfica. Miembro inferior de la Formación Toropí/ Yupoí (Figuras 1,2).

Descripción. CTES- PZ 3592 está representado por restos fragmentarios del caparazón y restos del pilar derecho (Figura 4). Entre ellos se preservó la placa periférica III derecha articulado con el pilar axilar derecho (Figuras 4A,B). De acuerdo a lo observado, la placa periférica III derecha posee forma rectangular del que se preservaron líneas de crecimiento rectangulares. También es posible observar el surco que divide los marginales 3 y 4 . En el pilar axilar derecho, se preservó la región anterior del escudo axilar en el que se observa seis lados (Figuras 4A,B). CTES- PZ 7491 está representado por parte de la placa costal muy silicificada de posición indeterminada (Figuras 4C,D). CTES- PZ 7490 (Figura 5) preserva restos fragmentarios de caparazón y cintura pélvica. Estos materiales se encuentran altamente silicificados e incluyen una placa neural, dos placas costales, dos periféricas y el acetábulo con la proyección del ilion (Figuras 5A-E,G). En la placa neural subhexagonal, de posición indeterminada, se desarrolla una giba vertebral de pendiente relativamente más angulosa que el ejemplar descripto por Zacarías et al. (2013), pero de menor espesor que el ejemplar descripto por Noriega et al. (2000) (Figura 5A). Las placas costales son rectangulares disminuyendo su ancho en la región distal. Estas placas también son de posición indeterminada, en el que solamente una preservó el surco interpleural (Figuras 5B,C). Las placas periféricas son subcuadrangulares, correspondientes al sector que articula caparazón y el plastrón, posiblemente correspondan a las placas periféricas IV y V (Figuras 5D,E). Presentan dos quillas, una de posición horizontal bien desarrollada debido a la forma en L levemente inclinada de la periférica (como es observado en las quillas de Ch. petersi y Ch. chilensis, ver Figura 5F) y otra que se desarrolla transversalmente en la superficie dorsal de la placa periférica (Figuras 5D,E). La quilla transversal está representada por la cresta que se forma en la posición del surco que delimita los escudos marginales. Dicha interpretación es producto de reconocer en esta quilla
A
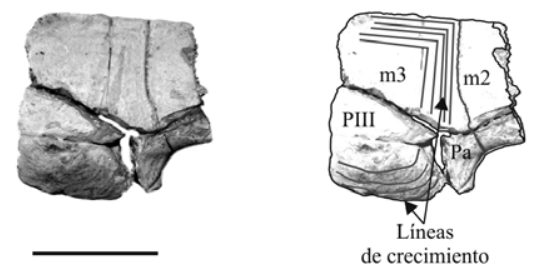

B
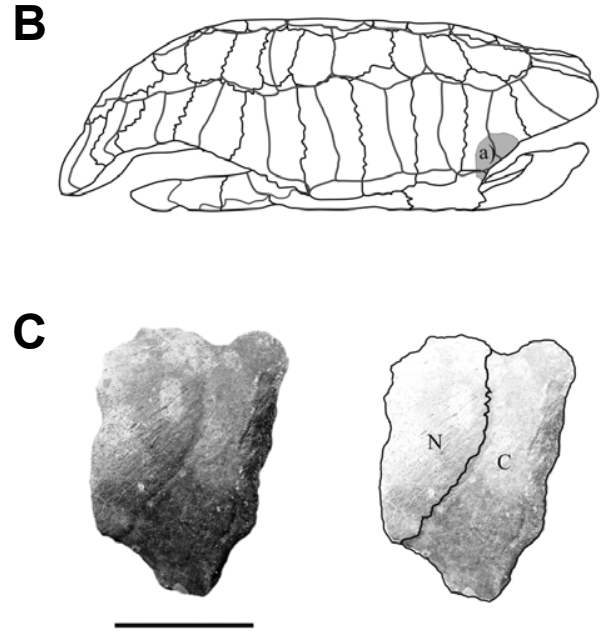

D

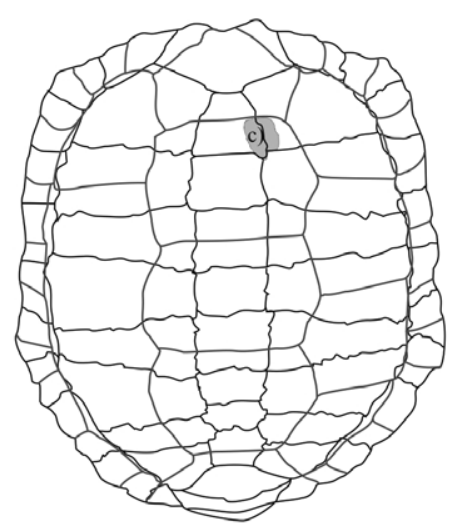

Figura 4. A, ejemplar CTES- PZ 3592, fragmento de caparazón, vista lateral, izquierda foto, derecha esquema. B, representación esquemática de una especie del grupo carbonaria, vista lateral, la mancha gris muestra el fragmento preservado. C, ejemplar CTES-PZ 7491, fragmento de caparazón, vista dorsal, izquierda foto, derecha esquema. D, ejemplar de Testudinidae, representación esquemática, vista dorsal, la mancha gris muestra el fragmento preservado. Abreviaturas: C, costal; N, neural; P, periférica; $\mathbf{m}$, marginal; $\mathbf{P a}$, pilar axilar. En los esquemas, líneas negras representan suturas (placas) y líneas grises representan surcos (escudos). Escalas: $A=50 \mathrm{~mm} ; C=25 \mathrm{~mm} ; B, D=$ sin escala.

Figure 4. A, specimen CTES- PZ 3592, carapace fragment, lateral view, left (picture), right (schematic representation). B, species of the carbonaria group, schematic representation, lateral view, the grey spot shows the part preserved. C, specimen CTES- PZ 7491, carapace fragment, dorsal view, left (picture), right (schematic representation). D, Testudinidae specimen, schematic representation, dorsal view, the grey area shows the part preserved. Abbreviations: C, costal; $\mathbf{N}$, neural; $\mathbf{P}$, peripherals; $\mathbf{m}$, marginal; $\mathbf{P a}$, axillar buttress. In the schemes, black lines represent sutures plates, and grey lines represent scutes. Scale bars: $A=50 \mathrm{~mm} ; C=25 \mathrm{~mm} ; B, D=$ without scale. 
transversal la típica forma lineal de las marcas de los surcos que delimitan escudos epidérmicos a diferencia de la forma en zigzag de las suturas. De la cintura pélvica, se preservó el acetábulo con una porción de la proyección iliaca, cuyo espesor disminuye distalmente, en dirección al caparazón (Figura 5G). Medidas. Tabla 1.

Determinación. La mayoría de los ejemplares aquí descriptos son asignables a la familia Testudinidae porque presentan placas del caparazón y pilar axilar grueso, placas costales arqueadas con expansión distal, gibas vertebrales, neurales y quillas en las placas periféricas. De acuerdo con distintos autores (Auffenberg, 1974; Pritchard \& Trebbau, 1984; De la Fuente, 1988; Ernst \& Barbour, 1989; Cabrera, 1998) estos caracteres están presentes en distintos taxones asignables a testudínidos. A su vez, los ejemplares CTES-PZ 7490 y CTES-PZ 7491 puede ser incluidos en el morfotipo "thick shelled giant tortoise" (Pritchard \& Trebbau, 1984) porque el gran tamaño de las placas espesas del caparazón y de la cintura pélvica,

Tabla 1. Medidas (mm) de los ejemplares referidos.

Table 1. Linear measurements $(\mathrm{mm})$ of referred specimens.

\begin{tabular}{ccc}
\hline Ejemplares & Elementos preservados & Medidas \\
\hline CTES- PZ 3592 & Fragmento de la PIII derecha & 70 \\
& Longitud recta & 7 \\
& Espesor & 15 \\
& Fragmento del pilar axilar & 23 \\
& Longitud recta & \\
& Espesor & 47
\end{tabular}

CTES- PZ 7491

Fragmento de la $\mathrm{N}$ de posición indeterminada

$\begin{array}{cc}\text { Longitud recta } & 80 \\ \text { Ancho } & 55 \\ \text { Espesor } & 25\end{array}$

CTES- PZ 7490

$\mathrm{N}$ de posición indeterminada

$\begin{array}{cc}\text { Longitud recta } & 165 \\ \text { Ancho } & 180 \\ \text { Espesor } & 89\end{array}$

$\mathrm{C}$ de posiciones indeterminadas

$\begin{array}{ll}\text { Longitud recta } & 380-440\end{array}$

Espesores 30

$P$ de posición indeterminada

Longitud lineal

CTES- PZ 7470

P del sector que articula con el plastrón

Longitud recta $\quad 35$

Ancho 25

P del sector caudal que articula con una porción de la C

Longitud recta 43

Ancho 28

P del sector caudal suelta

Longitud recta $\quad 27$

Epiplastrón

Longitud recta $\quad 55$

Ancho 95

Hipoplastrón

Longitud recta $\quad 45$

Ancho 48


Tabla 2. Especies (Chelonoidis nigra, Geochelone sulcata, Dipsochelys elephantina, Cylindraspis peltastes y Trachemys dorbigni) y coordenadas georeferenciadas disponibles en "Global biodiversity information faciliy (http://www.gbif.org/)", para la elaboración del mapa de distribución utilizando el programa Diva-Gis 7.50.

Table 2. Species (Chelonoidis nigra, Geochelone sulcata, Dipsochelys elephantina, Cylindraspis peltastes, and Trachemys dorbigni) and availables geo-referenciated data in "Global biodiversity information faciliy (http://www.gbif.org/)" used for the elaboration of the distribution map, by means of the software Diva-Gis 7.50.

\begin{tabular}{|c|c|c|}
\hline \multirow[t]{2}{*}{ Especies y ejemplares } & \multicolumn{2}{|c|}{ Coordenadas decimales } \\
\hline & Latitud & Longitud \\
\hline \multirow[t]{2}{*}{ Chelonoidis nigra } & -0.205059 & -90.72374 \\
\hline & -0.863233 & -91.12641 \\
\hline \multirow[t]{2}{*}{ Geochelone sulcata } & 16.016 & 16.5 \\
\hline & 16.25 & 33.75 \\
\hline Cylindraspis pelstates & -20.284677 & 57.604796 \\
\hline \multirow[t]{2}{*}{ Dipsochelys elephantina } & -18.774081 & 46.899201 \\
\hline & -23.466042 & 43.765705 \\
\hline \multirow[t]{2}{*}{ ?Chelonoidis australis } & -38.9599 & -61.7204 \\
\hline & -38.9599 & -61.7204 \\
\hline ?Chelonoidis gallardoi & -26.85777 & -66.07529 \\
\hline Chelonoidis lutzae & -28.5186 & -59.0584 \\
\hline \multirow[t]{3}{*}{ Ejemplares CTES 7490 y CTES 7491} & -28.988053 & -59.08807 \\
\hline & -28.987427 & -59.08852 \\
\hline & -28.522542 & -59.06021 \\
\hline \multirow[t]{9}{*}{ Trachemys dorbigni actuales } & -38.483497 & -62.88345 \\
\hline & -33.060386 & -58.53978 \\
\hline & -32.366191 & -58.22553 \\
\hline & -32.150089 & -58.13332 \\
\hline & -32.346933 & -57.87254 \\
\hline & -32.542881 & -55.77665 \\
\hline & -34.571413 & -56.35046 \\
\hline & -30.036007 & -51.20098 \\
\hline & -30.034017 & -51.23045 \\
\hline \multirow[t]{4}{*}{ Trachemys dorbigni fósiles } & -26.321491 & -59.37746 \\
\hline & -28.988053 & -59.08807 \\
\hline & -32.563093 & -60.76881 \\
\hline & -30.900743 & -62.80327 \\
\hline
\end{tabular}

indican que podrían tener un tamaño cercano a los $120 \mathrm{~cm}$ de longitud recta del caparazón. Dada la naturaleza fragmentaria de los materiales, no se encuentran ninguno de los cuatro caracteres diagnósticos del género Chelonoidis mencionados por Bour (1984). Sin embargo, la similitud morfológica existente con ejemplares asignados a Chelonoidis, permite referirlos de forma tentativa a dicho taxón. De modo particular, el ejemplar CTES-PZ 3592 presenta dos caracteres similares a Ch. carbonaria Spix, 1824, como ser el pilar axilar grueso con el escudo axilar expuesto y con forma poligonal. Por último, el ejemplar CTES-PZ 7490 posee costales con un ensanchamiento considerable hacia el extremo lateral, condición que se correspondería con la alternancia de costales anchas y estrechas que se observa en los testudinidos post-eocenos (Auffenberg, 1974). Adicionalmente, este material presenta una quilla horizontal desarrollada en la placa periférica dada la forma en $\mathrm{V}$, condición presente en el grupo chilensis (Auffenberg, 1971), aunque en este ejemplar la quilla está más marcada. Este ejemplar puede ser asignado a cf. Chelonoidis, por la presencia de la quilla horizontal en la placa periférica. Sin embargo, la presencia de una quilla transversal desarrollada en la superficie lateral dorsal de la periférica (que podría interpretarse como una cresta dispuesta en la posición del surco que separa dos escudos marginales) (Figuras 5D-E,J), tal vez sea una condición equivalente a la descripta por Williams (1950) para Chelonoidis cubensis (Leidy, 1868) (testudínido gigante recuperado del Pleistoceno de Cuba, América Central; Williams, 1950; Meylan \& Sterrer, 2000). Especie en la cual se observan crestas dispuestas en los surcos de algunas placas óseas del caparazón dorsal.

Familia EMYDIDAE Rafinesque, 1815

Trachemys Duméril \& Bibron, 1835

Especie tipo. Emys troosti Holbrook, 1836.

Trachemys cf. T. dorbigni

(Figura 6) 
A

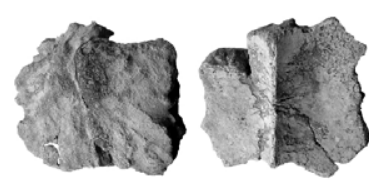

B

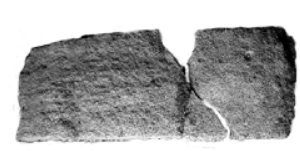

D
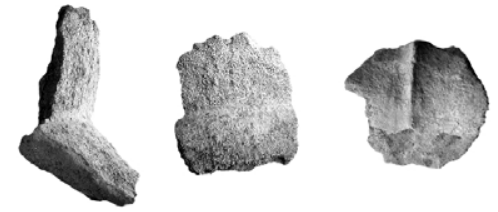

$\mathbf{E}$
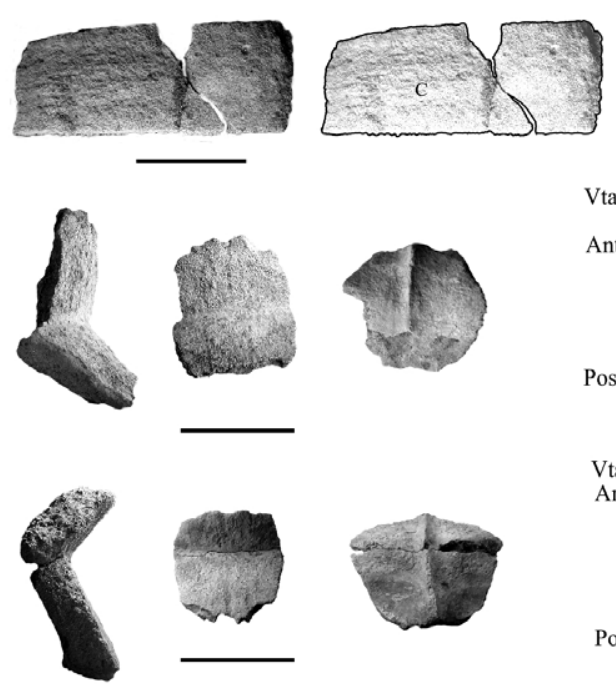

C
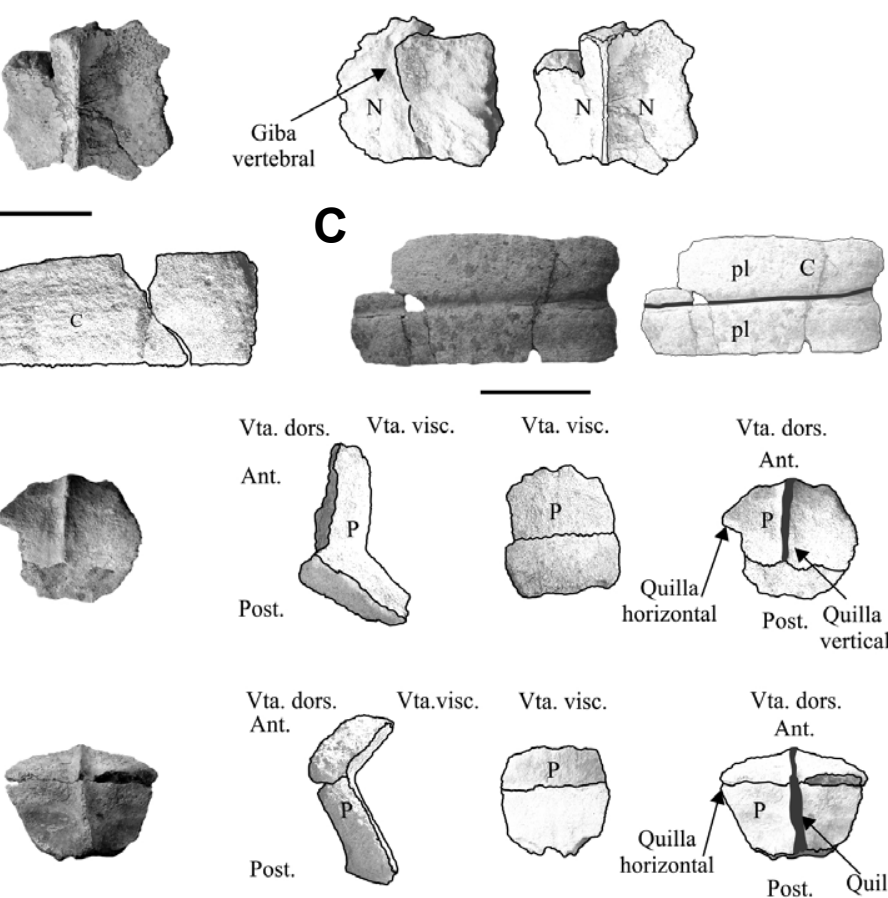

ta. dors.

Vta. dors. Vista Vta. visc.

$\mathbf{F}$

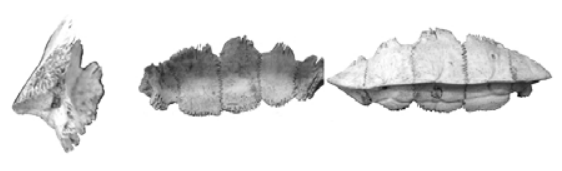

Ant. visceral
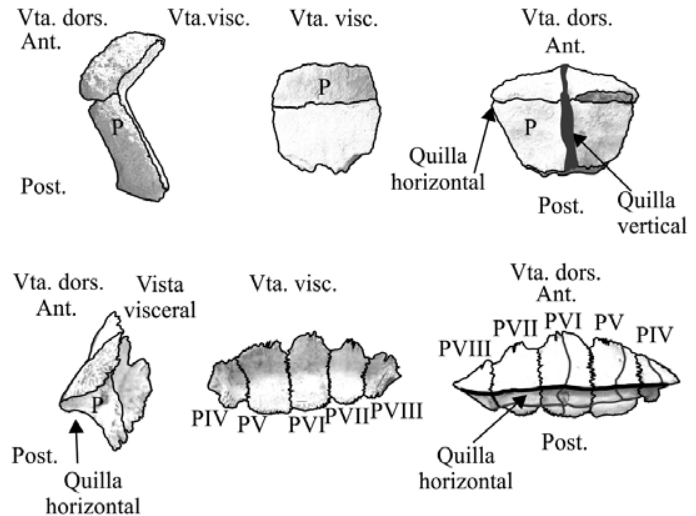

G
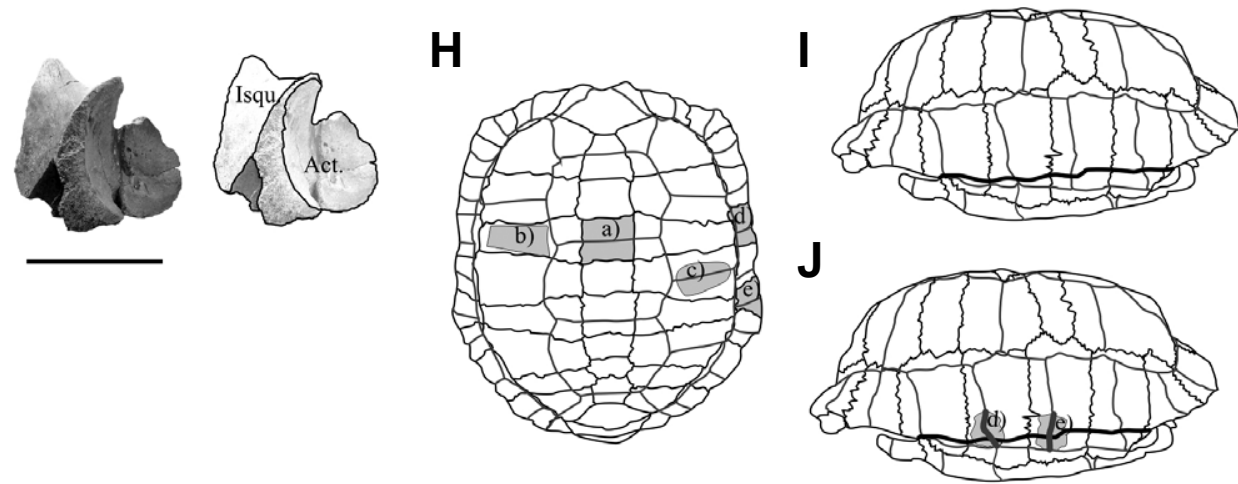

Figura 5. A-E, Ejemplar CTES- PZ 7490. A, izquierda vista dorsal y derecha vista visceral de las neurales, izquierda fotos; B-C, izquierda vista dorsal de costales indeterminadas, derecha esquema; $\mathbf{D}-\mathbf{E}$, periféricas de la región que articula con el puente. $\mathbf{F}$, periféricas de Chelonoidis chilensis Gray, para comparar con las periféricas del ejemplar CTES- PZ 7490, izquierda fotos, derecha esquemas; G, resto de cintura pélvica, izquierda foto, derecha esquema; H, Testudinidae (e.g. Ch. petersi Freiberg), vista dorsal del esquema de caparazón, las manchas grises representan el resto preservado. I, Testudinidae (e.g. Ch. chilensis), vista lateral del esquema de caparazón y plastrón. $\mathbf{J}$, Testudinidae, vista lateral del esquema de un caparazón y plastrón, las manchas grises representan el resto preservado. En los esquemas, líneas negras representan suturas (placas) y líneas grises representan surcos (escudos). Abreviaturas: Act., acetábulo; Ant., anterior; C, costal; dors., dorsal; Isqu., isquion; N., neural; $\mathbf{P}$, periférica; pl, pleural; post., posterior; visc.; visceral; vta., vista. Escalas: $A=100 \mathrm{~mm}$; $B, C=200 \mathrm{~mm} ; \mathrm{D}, \mathrm{E}=150 \mathrm{~mm} ; \mathrm{F}, \mathrm{G}=50 \mathrm{~mm} ; \mathrm{H}-\mathrm{J}=$ sin escala.

Figure 5. A-E, CTES- PZ 7490 specimen. A, dorsal view, left, visceral view of neurals plates, left (picture), right (drawing); B-C, dorsal view of indeterminate costals, left (picture), right (drawing); D-E, peripherals of region that articúlate with the bridge. F, Chelonoidis chilensis Gray peripherals, to compare than CTES- PZ 7490 peripherals, picture, left, drawing, right; G, fragment of pelvic girlde, left (picture), right (drawing); H, Testudinidae caparace scheme (e.g. Ch. petersi Freiberg), dorsal view, gray spot line represent preserved fragment. I, Testudinidae (e.g. Ch. chilensis), lateral view represents carapace and plastron scheme. J, Testudinidae, lateral view of carapace and plastron scheme, gray spots line represents preserved fragment. In the schematic representations, black lines shows plates and grey lines represents scutes. Abbreviations: Act., acetabulum; Ant., anterior; C, costal; Isqu., ischium; P, peripherals; pl, pleural; post., posterior; visc., visceral; vta., view. Scale bars: $A=100 \mathrm{~mm}$; B,C = 200 mm; $\mathrm{D}, \mathrm{E}=150 \mathrm{~mm} ; \mathrm{F}, \mathrm{G}=50 \mathrm{~mm} ; \mathrm{H}-\mathrm{J}=$ without scale. 
Material referido. Trachemys ef. T. dorbigni CTES- PZ 7470 (Figura 6).

Material de comparación. Apéndice 1.

Procedencia geográfica. Localidad de Santa Lucía (Figura 2B).

Procedencia estratigráfica. Miembro inferior de la Formación Toropí/ Yupoí (Figura 2B).

Descripción. CTES- PZ 7470 consiste en fragmentos muy silicificados de tres placas periféricas del sector derecho, lóbulo plastral anterior y parte del hipoplastrón (Figura 6). En general exhiben una coloración más oscura en los sectores correspondientes a los escudos, mientras que en sectores viscerales es más claro. Las placas periféricas tienen forma rectangular, una periférica corresponde al sector que articula con el plastrón y las otras del sector caudal porque poseen un borde libre. La periférica que articula con el plastrón tiene superficie rugosa, presenta una quilla y preserva surcos de los escudos marginales (Figura 6A). Las periféricas VIII y IX del sector caudal derecho posee articulado una porción de costal VI (Figura 6B). Estas periféricas poseen el escudo marginal 8 rectangular. La porción costal tiene un surco correspondiente a los escudos pleurales 3 y 4 (Figura 6B). La otra periférica recuperada, posee superficie estriada y preserva un surco correspondiente a una marginal (Figura 6C). El lóbulo plastral anterior está representado por el epiplastrón cuya superficie ventral es rugosa (Figura 6E). El borde libre del epiplastrón tiene forma convexa, en dicho elemento se preservaron los escudos gulares triangulares y parte del escudo humeral. Otro elemento del plastrón, es un fragmento derecho del hioplastrón con superficie ventral rugosa, en el se preservó el surco correspondiente al escudo abdominal (Figura 6F).

Medidas. Tabla 1.

Determinación. CTES- PZ 7470 presenta el lóbulo anterior del plastrón semejante al género Trachemys. La presencia de superficie rugosa en vista dorsal y ventral, tanto en restos de caparazón y plastrón, es un carácter diagnóstico de Trachemys (Vanzolini, 1995) y permiten confirmar la presencia del género para el Pleistoceno tardío de la Provincia de Corrientes. Las placas periféricas preservadas son aserradas, condición observada en T. dorbigni por Vanzolini (1995). Sin embargo, por el momento referiremos este ejemplar a $T$. cf. T. dorbigni hasta que se puedan recuperar ejemplares más completos.

\section{DISCUSIÓN Y CONCLUSIONES}

Pritchard \& Trebbau (1984) incluyen a las tortugas gigantes actuales y extintas de América del Sur en dos grandes categorías, las "thin giant tortoises" (tortugas de caparazón laminar) y las "thick shelled giant tortoises" (tortugas de caparazón espeso). Los testudínidos gigantes continentales corresponden a la categoría de "thick shelled giant tortoises" (sensu Pritchard \& Trebbau, 1984). Las tortugas gigantes agrupadas en la primera categoría, poseen representantes actuales de Sudamérica: Chelonoidis nigra de las islas Galápagos. A su vez, las tortugas agrupadas en la segunda categoría poseen representantes sudamericanas extintas tales como ?Ch. australis, ?Ch. gallardoi y Ch. lutzae, y los ejemplares gigantes aquí descriptos (CTES- PZ 7490 y 7491).

Desde una perspectiva paleoclimática, Broin (1984) propone que las tortugas podrían utilizarse prudentemente como indicadores climatológicos. Respecto de las tortugas terrestres, Auffenberg (1974) muestra una disminución del rango de distribución de Norte a Sur del género Geochelone (incluidas las tortugas terrestres gigantes) durante el Oligoceno-Mioceno y Plioceno-Pleistoceno. En este sentido, Schleich (1984) observa una congruencia entre el mapa de distribución actual de las especies del género Geochelone propuesta por Auffenberg (1974) con la región cálida del mapa climatológico de Geiger (1954). Para Schleich (1984) esta congruencia se debe a que, particularmente, las tortugas terrestres gigantes podrían ser buenos indicadores climáticos porque poseen mayor grado de termosensibilidad. Schleich (1984) justifica esta propuesta porque las tortugas terrestres gigantes son incapaces de hibernar y además deben evitar el sobrecalentamiento. A su vez, Mackay (1964) y Gerlach (2005) mencionan que las temperaturas cloacales para $\mathrm{Ch}$. nigra y Dipsochelys elephantina varían entre los $27,6^{\circ} \mathrm{C}$ a $34,5^{\circ} \mathrm{C}$, prefiriendo temperaturas ambientales que se encuentren dentro de ese rango durante la mayor parte del año.

Partiendo de la propuesta de Schleich (1984) hemos contrastado el mapa de distribución mundial de las tortugas terrestres gigantes actuales (Chelonoidis nigra, Geochelone sulcata, Dipsochelys elephantina, Cylindraspis peltastes) con el mapa climatológico elaborado por Kottek et al. (2006) (Figuras 7A,C) y observamos una congruencia de estas especies con la zona tropical (entre $-30^{\circ} \mathrm{y}+30^{\circ}$ ) y subtropical (entre $+30^{\circ} \mathrm{a}+40^{\circ}$ ).

Es de destacar que las tortugas terrestres gigantes son reptiles poiquilotermos, lo que condiciona su distribución mundial. Al margen de los fenómenos de insularidad, los reptiles pueden llegar a tamaños muy grandes en las zonas tropicales porque las condiciones climáticas son favorables (Wagner, 1993). De esta manera en América del Sur se observan tortugas de pequeño a mediano tamaño en zonas subtropicales (Chelonoidis chilensis y Ch. petersi), tortugas de gran tamaño en zonas subtropicales y tropicales (Ch. carbonaria y $C h$. denticulata) y gigantes en la zona tropical (Ch. nigra).

En este subcontinente se registran tres especies cenozoicas de tortuga terrestres gigantes continentales tales como ?Ch. australis, ?Ch. gallardoi y Ch. lutzae. Si bien las tres especies de tortugas terrestres gigantes continentales corresponden a distintas edades geológicas, la información geológica (Marshall \& Patterson, 1981; Zavala \& Navarro, 1993; Iriondo 2010), paleoclimática (Le Roux, 2012a,b), palinológica (Barreda et al., 2007) y paleontológica (Cione et al., 2007) de las unidades de donde fueron halladas estas especies, demostraría que las condiciones climáticas en que vivieron estas tortugas podrían haber sido cálidas. De este modo, y de acuerdo a las estimaciones paleoambientales de las formaciones Andalhualá (Barreda et al., 2007), Monte Hermoso (Zavala \& Navarro, 1993) y Toropí/ Yupoí (ver contexto geológico, estratigráfico y paleoambiental), ?Ch. gallardoi, ?Ch. australis y Ch. lutzae habitaron la gran región 
A
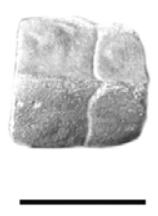

B
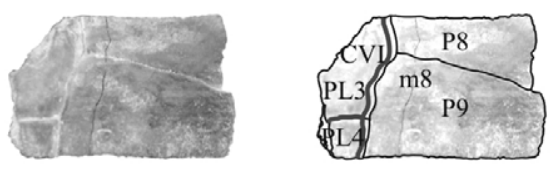

C

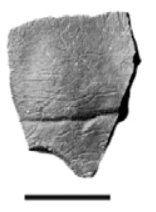

E
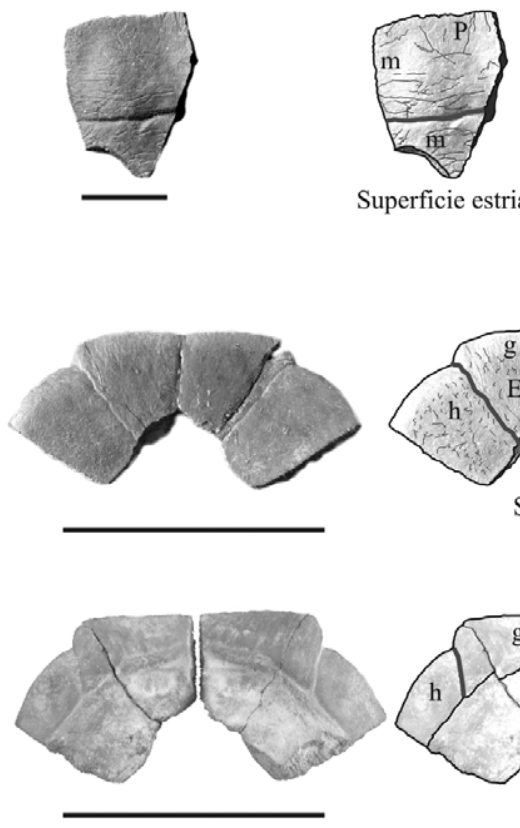

Superficie estriada
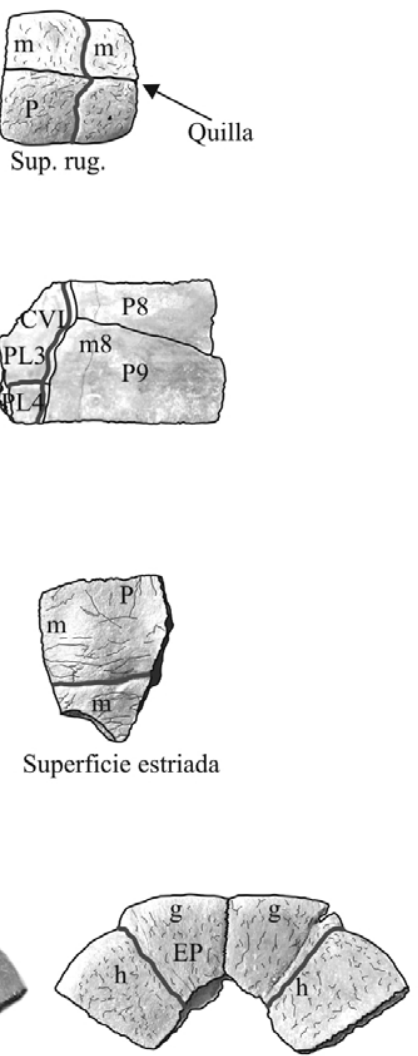

Sup. rug.

$\mathbf{F}$

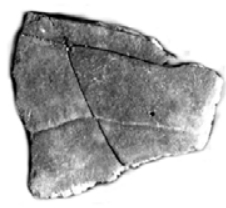

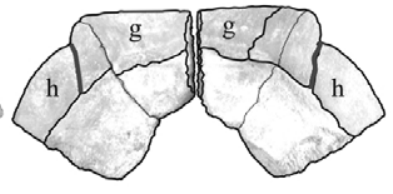

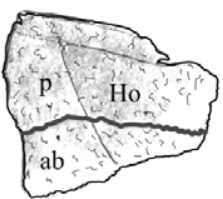

Sup. rug.
D

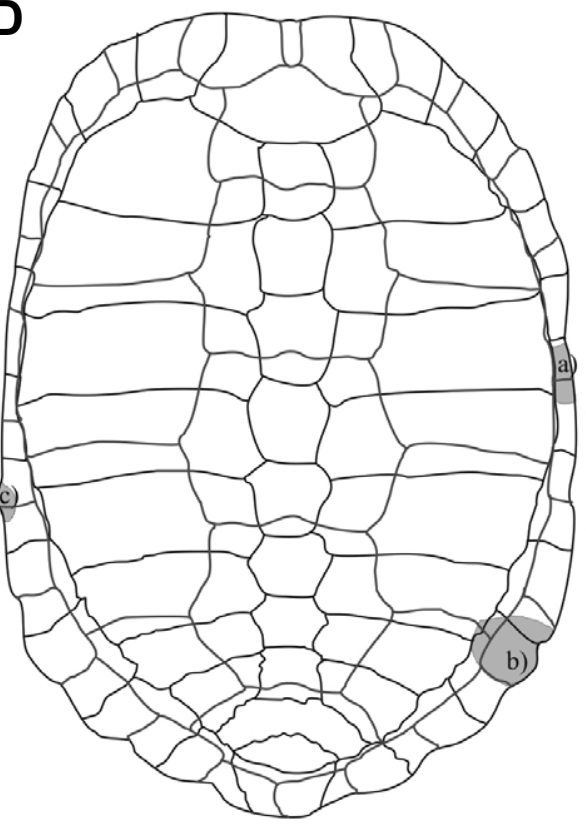

G

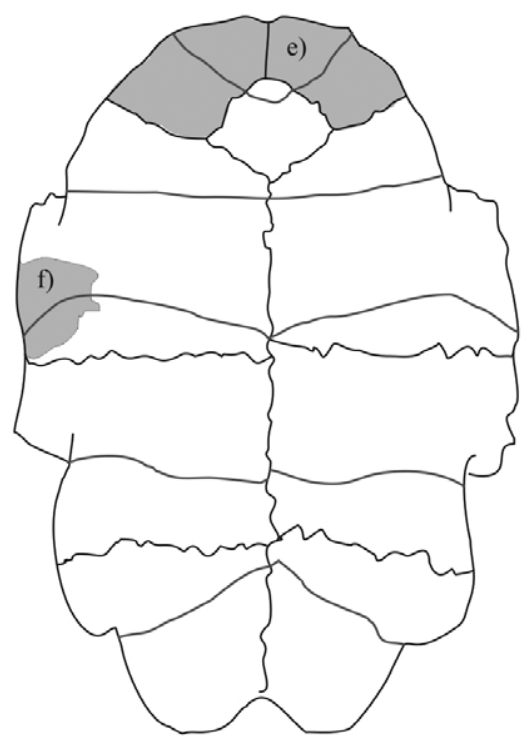

Figura 6. A-C, Ejemplar CTES-PZ 7470. A, periférica correspondiente a la región que articula con el plastrón izquierda, foto, derecha esquema; B, periféricas de la región caudal que articulan con una porción de caudal, izquierda foto, derecha esquema; C, periférica de posición indeterminada, izquierda foto, derecha esquema. D-G, Trachemys dorbigni Duméril \& Bibron. D, vista dorsal del esquema de caparazón, las manchas grises representan los restos preservados; E, fragmento del lóbulo plastral anterior, arriba vista dorsal, abajo vista visceral, izquierda foto, derecha esquema; F, fragmento de porción plastral del hioplastrón, izquierda foto, derecha esquema; G, vista ventral del esquema del plastrón de $T$. dorbigni las manchas grises representan los restos preservados. En los esquemas, líneas negras representan suturas (placas) y líneas grises representan surcos (escudos). Abreviaturas: Ab, abdominal; C, costal; EP, Epiplastrón; g, gular; h, humeral; Ho, Hioplastrón; m, marginal; P, periférica; $\mathbf{p}$, pectoral; PL, pleural; rug., rugosa; sup., superficie. Escalas: $A, C=10 \mathrm{~mm} ; B, F=30 \mathrm{~mm} ; E=40 \mathrm{~mm} ; D, G=\sin$ escala.

Figure 6. A-C, CTES-PZ 7470 specimen. A, peripherals corresponding to the region articulating with the plastron; left (picture), right (schematic representation); B, peripherals of the caudal region that articulate with one of the caudal portion picture, left, scheme, right; $\mathbf{C}$, peripherals of indeterminate location picture, left, scheme, right. D-G, Trachemys dorbigni Duméril \& Bibron. D, schematic dorsal view of the carapace, the grey area represents the preserved rest; $\mathbf{E}$, fragment of the anterior plastral, dorsal view, up, visceral view, down, picture, left, scheme, right; $\mathbf{F}$, preserved fragment of hyoplastral portion, picture, left, scheme, right; $\mathbf{G}$, ventral view of $T$. dorbigni plastron scheme, gray line represents preserved fragment. In the schematic representations, black lines shows plates and grey lines represents scutes. Abbreviations: C, costal; EP, epiplastron; g, gular; $\mathbf{h}$, humeral; Ho, hyoplastron; $\mathbf{m}$, marginal; $\mathbf{P}$, peripherals; $\mathbf{p}$, pectoral; rug., rugose; sup., surface. Scale bars: $A, C=10 \mathrm{~mm} ; B, F=30 \mathrm{~mm} ; E=40 \mathrm{~mm} ; D, G=$ without scale. 
Neotropical más relacionado con la provincia fitogeográfica chaqueña, donde ?Ch. gallardoi, ?Ch. australis podrían haber estado más vinculados a ambientes más secos y Ch. lutzae podría haber estado más vinculado a ambientes más húmedos.

Por consiguiente, la evidencia nos lleva a concluir que la presencia de tortugas terrestres gigantes extintas [?Chelonoidis australis, ?Ch. gallardoi y Ch. lutzae (sensu Zacarías et al., 2013)] en el continente Sudamericano podría coincidir con condiciones climáticas cálidas para los lapsos que representan las unidades litoestratigráficas respectivas de procedencia de estas tortugas.

Por otro lado, el ejemplar PZ-CTES 7470 representa la primera cita de Trachemys cf. T. dorbigni para el Pleistoceno tardío de la Provincia de Corrientes. T. dorbigni habita en la actualidad en ambientes constituidos por sistemas de lagunas poco profundas o en ríos y canales menores con menor energía acompañados de selva en galería (Cabrera, 1998). De acuerdo al mapa de distribución de T. dorbigni y el mapa climatológico de Kottek et al. (2006), es posible observar que el ambiente de esta especie se caracteriza por ser muy húmedo, con veranos cálidos y temperaturas templadas (Figura 7B). Rocha (2005) menciona que la temperatura cloacal de T. dorbgni varían entre $24^{\circ} \mathrm{C}$ a $29^{\circ} \mathrm{C}$, en aguas donde las temperaturas oscilaban entre $13,5^{\circ} \mathrm{C}$ en invierno y $32^{\circ} \mathrm{C}$ en verano. De esta manera, se puede inferir que los especímenes extintos asignados a Trachemys habitaron durante el Pleistoceno tardío en paleoambientes semejantes en el área de Santa Lucía, sugiriendo la existencia de condiciones climáticas cálidas y húmedas para esta región, en congruencia con la información geológica, paleobotánica y paleofaunística disponible para la Formación Toropí/Yupoí (Herbst, 1971; Herbst \& Santa Cruz, 1985; Scillato Yané et al., 1998; Lutz \& Gallego, 2001; Albino
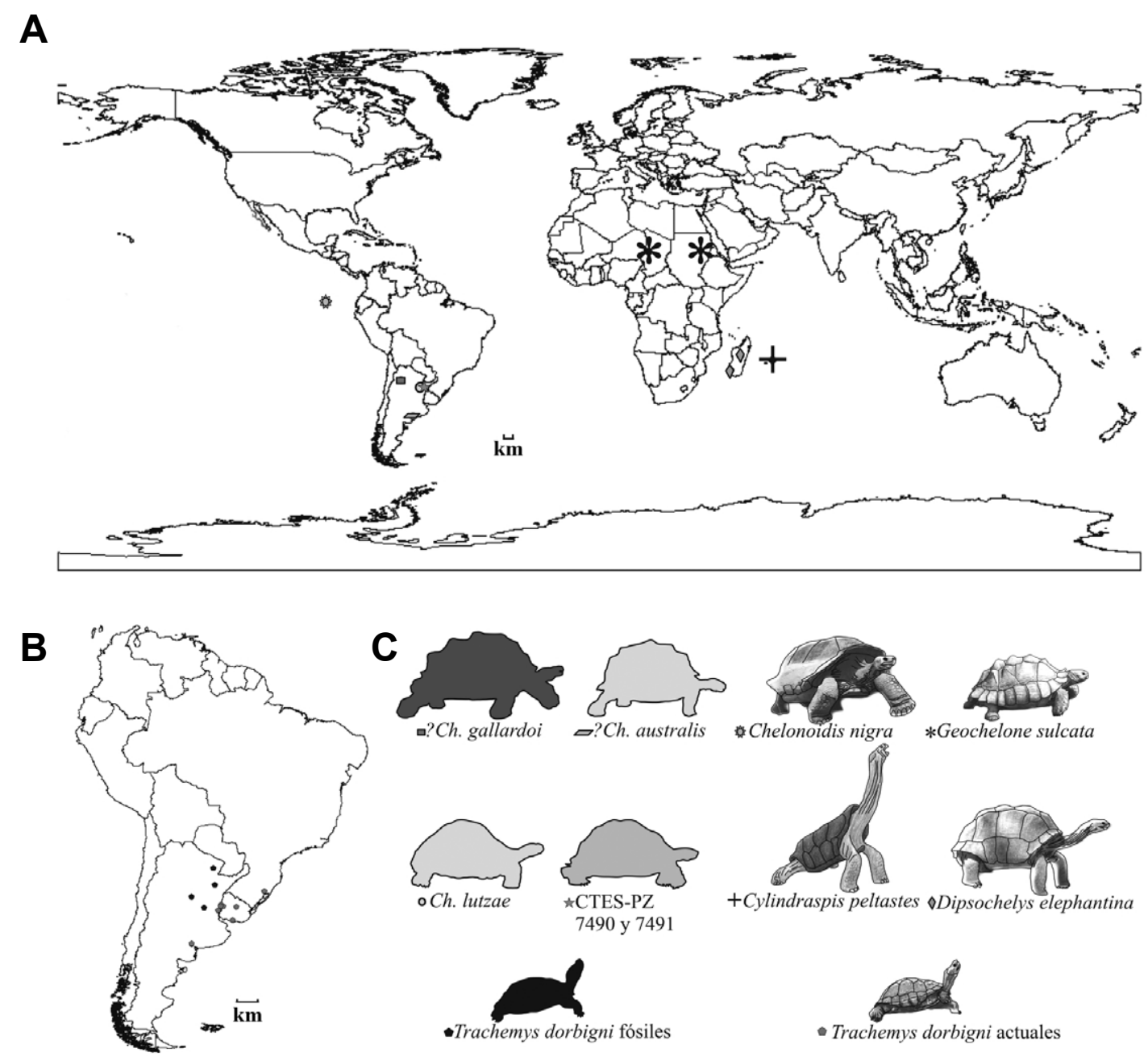

Figura 7. A, mapa que muestra la distribución de los Testudinidae gigantes actuales y los fósiles de América del Sur. B, mapa que muestra la distribución de Trachemys dorbigni. C, referencias. Escalas $=0-400 \mathrm{~km}$. Los mapas se obtuvieron a partir del Diva-Gis 7.50 , utilizando los datos georeferenciados de las especies Chelonoidis nigra, Dipsochelys elephantina, Cylindraspis peltastes y Trachemys dorbigni disponibles en Global biodiversity information facility (http://www.gbif.org/), (ver Tabla 2).

Figure 7. A, map showing the geographic distribution of extinct and extant South American giant Testudinidae. B, map showing the distribution of Trachemys dorbigni. C, references. Scale bars $=0-400 \mathrm{~km}$. The maps were obtained from the Diva-Gis 7.50 , using geo-referenciated data of the species Chelonoidis nigra, Dipsochelys elephantina, Cylindraspis peltastes y Trachemys dorbigni, which are available in Global biodiversity information facility (http://www.gbif.org/), (see Table 2). 
\& Carlini, 2008; Carlini et al., 2008; Iriondo \& Krohling, 2008; Tonni, 2009; Francia et al., 2009, 2012b, Alcaraz, 2010; Iriondo, 2010; Erra et al., 2010, 2013).

Finalmente, las inferencias paleoclimáticas y paleoambientales de los ejemplares aquí presentados son congruentes con las hipótesis que establecen un paleoclima cálido y húmedo [hipótesis (3a); (1b); (2b)] para la Formación Toropí/Yupoí. Ya que solamente en estas condiciones paleoclimáticas del Pleistoceno tardío en la Formación Toropí/ Yupoí, pudieron haber sido favorables para los especímenes terrestres gigantes de cf. Chelonoidis y especímenes dulceacuícolas de Trachemys.

\section{AGRADECIMIENTOS}

Los autores desean expresar su agradecimiento al P. Cuaranta, quien encontró y proporcionó los ejemplares de cf. Chelonoidis gigantes y de Trachemys $\mathrm{cf}$. T. dorbigni en la localidad de Santa Lucía. A la L.S. Morton (CECOALCONICET, Corrientes) por proporcionarnos el ejemplar de cf. Chelonoidis hallado en Bella Vista, Arroyo Toropí. A M. Iriondo (Universidad Nacional del Litoral, Santa Fé), O. Orfeo (CECOAL-CONICET, Corrientes) y J.M.D. Gómez (Instituto de Biología y Geología del Noa, Salta) por sus valiosos comentarios. Al F. Rubel (Grupo de Biometeorología y Epidemológica Matemática del Instituto de Veterinaria y Salud Pública, de la Universidad de Veterinaria y Medicina, Vienna, Austria) por proporcionar el mapa mundial climatológico. También a los dos revisores (J. Sterli y un revisor anónimo) y al editor (F. Abdala), quienes con sus observaciones y sugerencias contribuyeron a mejorar este manuscrito. Esta contribución fue parcialmente realizada y financiada en el marco de las becas otorgadas por la Universidad Nacional del Nordeste y CONICET (Pregrado SGCyT-UNNE períodos 2009-2010, 2010-2012 y CONICET postgrado tipo I período 2012-2015) obtenidas por el G.G. Zacarías y el PIP 00795 (CONICET).

\section{REFERENCIAS}

Albino, M.A. \& Carlini, A.A. 2008. First Record of Boa constrictor (Serpentes, Boidae) in the Quaternary of South America. Journal of Herpetology, 42:82-88. doi:10.1670/07-124r1.1

Alcaraz, M.E. 2010. Sistemática de los Cérvidos (Mammalia, Arctiodáctyla) del Pleistoceno de Áreas Extrandinas de Argentina. Universidad Nacional de La Plata, Tesis doctoral, $317 \mathrm{p}$.

Álvarez, B.B. 1974. Los mamíferos fósiles del Cuaternario de Arroyo Toropí, Corrientes, Argentina. Ameghiniana, 11:295-311.

Auffenberg, W. 1971. A new fossil tortoise, with remarks on the origin of South American Testudinines. Copeia, 1:106-117.

Auffenberg, W. 1974. Cheklist of fossil land tortoise (Testudinidae). Bulletin of the Florida State Museum, Biological Science, 18:121-251.

Batsch, A.J.G.C. 1788. Versuch einer Anleitung, zur Kenntniß und Geschichte der Thiere und Mineralien. Jena, Akademische Buchhandlung, $528 \mathrm{p}$.

Barreda, V. et al. 2007. Diversificación y cambios de las angiospermas durante el Neógeno en Argentina. Buenos Aires,
Asociación Paleontológica Argentina, p. 173-191 (Publicación Especial 11).

Bour, R. 1984. Les tortues terrestres géants des îles de l'Océan Indien Occidental: données géographiques, taxinomiques et phylogénetiques. Stvdia Palaeocheloniologica, 1:17-76.

Broin, F. 1984. Róle des tortues comme indicateurs de climat. Stvdia Geologica Salmanticensia, 1:99-103.

Broin, F. \& de la Fuente, M.S. 1993. Les tortues fossiles d'Argentine: synthèse. Annales de Paléontologie, 79:169-232.

Cabrera, M.R. 1998. Las Tortugas Continentales de Sudamérica Austral. Córdoba, Talleres gráficos BR Copias, 115 p.

Cabrera, M. \& Luna, A.C. 2011. Presencia de Trachemys Agassiz, 1857 (Testudines, Emydidae) en el Pleistoceno tardío del centro de la Argentina. Cuadernos Herpetológicos, 25:36-41.

Carlini, A.A.; Zurita, A.E.; Gasparini, G. \& Noriega, J.I. 2004. Los mamíferos del Pleistoceno de la Mesopotamia argentina y su relación con los del Centro Norte de la Argentina, Paraguay y Sur de Bolivia, y los del Sur de Brasil y Oeste de Uruguay: paleobiogeografía y paleoambientes. In: F.G. Aceñolaza (coord.) Temas de la biodiversidad del litoral fluvial argentino, Tucumán, INSUGEO, p. 83-90 (Miscelánea 12).

Carlini, A.A.; Zurita, A.E. \& Miño-Boilini, A.R. 2008. Reseña paleobiogeográfica de los Xenarthra (Mammalia) del Pleistoceno tardio de la región Mesopotámica (Argentina), Tucumán, INSUGEO, p. 259-270 (Miscelánea 17).

Cione, L.A. \& Tonni, E.P. 2005. Bioestratigrafía basada en mamíferos del Cenozoico superior de la provincia de Buenos Aires, Argentina. In: CONGRESO GEOLÓGICO ARGENTINO, 16, 2005. Actas, La Plata, APA, p. 183-200.

Cione, A.L. et al. 2007. Mamíferos continentales del Mioceno tardio a la actualidad en la Argentina: cincuenta años de estudios. Buenos Aires, Asociación Paleontológica Argentina, p. 257-278 (Publicación Especial 11).

De la Fuente, M.S. 1988. Las tortugas Chelidae (Pleurodira) y Testudinidae (Cryptodira) del Cenozoico argentino. Universidad Nacional de La Plata, Tesis doctoral, $270 \mathrm{p}$.

De la Fuente, M.S. 1994. Descripción de nuevos especímenes y relaciones filogenéticas de Chelonoidis gringorum (Chelonii: Testudinidae) del Mioceno temprano de Patagonia central. Studia Geologica Salmanticensia, 30:107-131.

De la Fuente, M.S. 1997. Las tortugas terrestres gigantes del Mioceno tardío-Plioceno del territorio argentino. Stvdia Geologica Salmanticensia, 33:91-120.

De la Fuente, M.S. 1999. A review of the Pleistocene Reptiles of Argentina: taxonomic and palaeoenvironmental considerations. Quaternary of South America and Antarctic Peninsula, 12:111-138.

De la Fuente, M.S.; Noriega, J.I. \& Piña, C.I. 2002. Trachemys dorbigni (Duméril y Bibron, 1835) (Cryptodira: Emydidae) en el Pleistoceno tardío de la provincia de Entre Ríos, Argentina. Cuadernos de Herpetología, 16:65-72.

Dumeril, A.M.C. \& Bibron, G. 1835. Erpetologie generale ou histoire naturalle complete des Reptiles, 2. Paris, Librarie Encyclopedique de Rote, $680 \mathrm{p}$.

Ernst, C.H. \& Barbour, R.W. 1989. Turtles of the world. Washington D.C., Smithsonian Institution Press, 313 p.

Erra, G.; Lutz, A.I.; Zurita, A.E.; Osterrieth, M.L. \& Francia, A. 2010. Fitolitos en termiteros fósiles del Pleistoceno tardío de Corrientes, Argentina. In: SIMPÓSIO BRASILEIRO DE PALEOBOTÂNICA E PALINOLOGIA, 13, 2000. Anais, Salvador, p. 172-173.

Erra, G.M.; Osterrieth, M.; Zurita, A.E.; Francia, A. \& Carlini, A.E. 2013. Paleoenvironment in the Toropí Formation (Upper 
Pleistocene), Corrientes province (Mesopotamia region, Argentina) phylolithic approach. Quaternary International, 287:73-82. doi:10.1016/j.quaint.2012.08.2110

Fernández, M.S. 1988. Las Testudinidae (Reptilia: Chelonii) argentinas: osteología, sistemática y distribución geográfica. Universidad Nacional de La Plata, Tesis doctoral, $276 \mathrm{p}$.

Fitzinger, L. 1826. Neue Classification der Reptilien nach ihren Natürlichen Verwandtschaften nebst einer VerwandtschaftsTafel und einem Verzeichnisse der Reptilien-Sammlung des Zoologischen Museum zu Wien. Vienna, K.K. Hof-Naturaliencabinet, $66 \mathrm{p}$.

Fitzinger, L. 1835. Entwurf einer systematischen Anordnung der Schildkröten nach den Gründsatzen der natürlichen Methode. Annalen des Wiener Museum der Naturgeschichte, 1:105-128.

Francia, A.; Carlini, A.A. \& Zurita, A.E. 2009. Novedades acerca de Mamíferos cuaternarios del Arroyo Toropí, Corrientes: sus implicancias biogeográficas. In: COMUNICACIONES CIENTÍFICAS Y TECNOLÓGICAS, 2009. Libro de resúmenes on-line, Chaco, versión sin paginación CB-29 (www.unne.edu. ar).

Francia A.; Carlini, A.A.; Zurita, A.E.; Miño-Boilini, A.R. \& Kruck, W. 2012a. Cronología de las unidades litoestratigráficas aflorantes en el Arroyo Toropí, Provincia de Corrientes y los registros paleofaunísticos. In: REUNIÓN DE COMUNICACIONES CIENTÍFICAS Y TECNOLÓGICAS, 2012. Libro de resúmenes on-line, Corrientes, versión sin paginación CB-033 (www.unne. edu.ar).

Francia, A.; Carlini, A.A.; Zurita, A.E. \& Verzi, D. 2012b. Galea (Rodentia, Caviidae) in the late Pleistocene of Corrientes province (Argentina): taxonomic and paleobiogeographic implications. Neues Jahrbuch fur Geolgie und Paläontologie Abhandlungen, 266:173-184. doi:10.1127/0077-7749/2012/0292

Freiberg, M.A. 1973. Dos nuevas tortugas terrestres de Argentina. Boletín de la Sociedad Biológica Concepción, 46:81-93.

Fritz U.; Alcalde L.; Vargas-Ramirez M.; Goode E.V.; FabiousTuroblin, D.U. \& Praschag, P. 2012a. Northern genetic richness and southern purity, but just one species in Chelonoidis complex. Zoological Scripta, 41:220-232. doi:10.1111/j.14636409.2012.00533.x

Fritz, U.; Stuckas, H.; Vargas-Ramírez, M.; Hundsdörfer, A.K.; Maran, J. \& Päckert, M. 2012b. Molecular phylogeny of Central and South American slider turtles: implications for biogeography and systematics (Testudines: Emydidae: Trachemys). Journal of Zoological Systematics and Evolutionary Research, 50:125-136. doi:10.1111/j.1439-0469.2011.00647.x

Geiger, R. 1954. Landolt-Börnstein - Zahlenwerte und Funktionen aus Physik, Chemie, Astronomie, Geophysik und Technik. In: W. Köppen (ed.) Klassifikation der Klimate, Springer, p. 603-607.

Gerlach, J. 2005. Thermoregulation in captive Indian Ocean Giant tortoises. Chelonoian Consevation and Biology, 4:924-928.

Gray, J.E. 1825. A synopsis of the genera of reptiles and Amphibia, with a description of some new species. Annals of Philosophy, 10:193-217.

Gray, J.E. 1870. Notes on tortoises in the British Museum, with descriptions of some new species. Proceedings of the Zoological Society of London, 1870:653-659.

Herbst, R. 1971. Esquema estratigráfico de la provincia de Corrientes, República Argentina. Revista de la Asociación Geológica Argentina, 26:221-243.
Herbst, R. \& Álvarez, B.B. 1974. Nota sobre dos formaciones del Cuartario de Corrientes, República Argentina. Revista FaCENA, 1:7-17.

Herbst, R. \& Santa Cruz, J. 1985. Mapa litoestratigráfico de la provincia de Corrientes. D'Orbignyana, 2:1-69.

Husby, E.C. 2009. Ecophysiology and Biomechanics of Equisetum giganteum in South America. Florida International University, Ph.D. Thesis, 181p.

Iriondo, M.H. 1973. Mineralogía de la Formación Yupoí. Revista de la Asociación de Ciencias Naturales del Litoral, 4:87-96.

Iriondo, M.H. 2007. Introducción a la Geología. Córdoba, Editorial Brujas, $236 \mathrm{p}$.

Iriondo, M.H. 2010. Geología del Cuaternario en Argentina. Corrientes, Editorial Moglia, 437p.

Iriondo, M.H. \& Kröhling D.M. 2008. Cambios ambientales en la cuenca del río Uruguay desde dos millones de años hasta el presente. Santa Fe, Universidad Nacional del Litoral, 350 p.

Joyce W.G.; Parham J.F. \& Gauthier J.A. 2004. Developing a protocol for the conversion of rank-based taxon names to phylogenetically defined clade names, as exemplified by turtles. Journal of Paleontology, 78:989-1013. doi:10.1666/00223360(2004)078<0989:DAPFTC > 2.0.CO;2

Kottek, M.; Grieser, J.; Beck, C.; Rudolf, B. \& Rubel, F. 2006. World map of the Köppen-Geiger climate classification updated. Meteorologische Zeitschrift, 15:259-263. doi:10.1127/09412948/2006/0130

Le Roux, J.P. 2012a. A review of Tertiary climate changes in southern South America and the Antarctic Peninsula. Part1: Oceanic conditions. Sedimentary Geology, 247-248:1-20. doi:10.1016/j. sedgeo.2011.12.014

Le Roux, J.P. 2012b. A review of Tertiary climate changes in southern South America and the Antarctic Peninsula. Part2: Oceanic conditions. Sedimentary Geology, 247-248:21-38. doi:10.1016/j. sedgeo.2011.12.001

Lutz, A. \& Gallego, O. 2001. Nuevos hallazgos fosilíferos (vegetales e icnofósiles) en el cuaternario de la provincia de Corrientes. Ameghiniana, 38:36R.

Mackay, R.S. 1964. Galapagos tortoise and marine iguana deep body temperatures measured by radio telemetry. Nature, 204:355-358. doi:10.1038/204355a0

Marshall, L.G. \& Patterson, B. 1981. Geology and geochronology of mammal bearing Tertiary of Valle de Santa María and Rio Corral Quemado, Catamarca province, Argentina. Fieldiana Geology, 9:1-80.

Martínez R, J.I. 2009. La historia cenozoica del fenómeno de El Niño. Revista Académica Colombia, 33:491-511.

Meerbeeck, C.J.; Renssen, H. \& Roche, D.M. 2009. How did marine isotope stage 3 and Last Glacial Maximum climates differ? Perspectives from equilibrium simulations. Climate of the Past, 5:33-51.

Meylan, P.A. \& Sterrer, W. 2000. Hesperotestudo (Testudines: Testudinidae) from the Pleistocene of Bermuda, with comments on the phylogenetic position of the genus. Zoological Journal of the Linnean Society, 128:51-76. doi:10.1111/j.1096-3642.2000. tb00649.x

Noriega, J.; Manzano, A.; de la Fuente, M.S. \& Tonni, E. 2000. Un Testudininae gigante (Chelonii: Cryptodira) del Pleistoceno de la Provincia de Corrientes, Argentina. Ameghiniana, 37:321-326.

Orfeo, O. \& M., Iriondo. 2010. The megafan of the Paraná River from the Pleistocene to the Present. In: INTERNATIONAL SEDIMENTOLOGICAL CONGRESS, 18, 2010. Abstracts, Mendoza, ISA, p. 663.

Pascual, R.; Ortega Hinojosa, E.J.; Gondar, D. \& Tonni, E.P. 1965. Las edades del Cenozoico mamalífero de la Argentina 
con especial atención a aquellas del territorio bonaerense. In: COMISIÓN DE INVESTIGACIONES CIENTÍFICAS DE LA PROVINCIA DE BUENOS AIRES, 6, 1965. Anales, La Plata, p. 165-194.

Pritchard, P.C. \& Trebbau, P. 1984. The Turtles of Venezuela. Contribution in Herpetology, 12:1-403.

Rafinesque, C.S. 1815. Analyse de la nature ou Tableau de l'Univers et des corps organisés. Palermo, Jean Barravecchia, 224 p.

Richard, E. 2009. Tortugas de Argentina (situación regional, nacional, sudamericana). Documento de trabajo jornadas regionales sobre estado de conservación de la fauna y flora amenazada. In: E. Richard \& C.A. Nigro (eds.) Cátedra de Medicina Veterinaria, Manejo y Conservación de Fauna Silvestre: Syllabus, Facultad de Ciencias Veterinarias, Universidad Nacional de Rosario, 71 p.

Rocha, D.F.N.B. 2005. Biologia termal das tartarugas Trachemys dorbigni (Duméril \& Bibron, 1835) e a Trachemys scripta elegans (Wied, 1839) dos lagos de Porto Alegre, RS, Brasil (Testudines, Emididae). Programa de Pós-Graduação em Biologia Animal, Universidade Federal o Rio Grande do Sul, Dissertação de Mestrado, 68 p.

Schleich, H.H. 1984. Neogene Testudines of Germany, their stratigraphical and ecological evaluation. Stvdia Geologica Salmanticensia, 1:249-267.

Scillato-Yané, G.J.; Tonni, E.P.; Carlini, A.A. \& Noriega, J.I. 1998. Nuevos hallazgos de mamíferos del Cuaternario en el Arroyo Toropí, Corrientes, Argentina. Aspectos bioestratigráficos, paleoambientales y paleozoogeograficos. In: CONGRESO LATINOAMERICANO DE GEOLOGÍA, 10 Y CONGRESO NACIONAL DE GEOLOGÍA ECONÓMICA, 6, 1998. Actas, Buenos Aires, p. 263-268.

Tonni, E.P. 2009. Las unidades portadoras de vertebrados del Cuaternario de las regiones Mesopotámica y Pampeana Oriental de la Argentina: ensayo de correlación. In: A.M. Ribeiro; S.G. Bauermann \& C.S. Scherer (eds.) Quaternário do RS: integrando conhecimentos, Sociedade Brasileira de Paleontologia, p. 57-66 (Monografias 1).
Van Dijk, P.P.; Iverson, J.B.; Bradley Shaffer, H.; Bour, R. \& Rhodin A.G.J. 2011. Turtles of the World, 2011 update: annotated checklist of taxonomy, synonymy, distribution, and conservation status. In: A.G.J. Rhodin; P. Pritchard; P.P. van Dijk; R.A. Saumure; K.A. Buhlmann; J.B. Iverson \& R.A. Mittermeier (eds.) Conservation biology of freshwater turtles and tortoises. A compilation project of the IUCNISSC tortoise and freshwater, Ross, Turtle Specialist Group, p. 165-242 (Chelonian Research Monographs 5).

Vanzolini, P.E. 1995. A new species of turtle, genus Trachemys, from the State of Maranaho, Brazil (Testudines, Emydidae). Revista Brasileira de Biologia, 55:111-125.

Wagner, C. 1993. Entender la ecología, cómo funciona el equilibrio natural, sus puntos débiles y la influencia humana. Barcelona, Blume, $242 \mathrm{p}$.

Williams, E. 1950. Testudo cubensis and the evolution of Western Hemisphere tortoises. Bulletin of the American Museum of Natural History, 95:7-34.

Zacarías, G.G.; de la Fuente, M.S.; Fernandez, M.S. \& Zurita, A.E. 2013. Nueva especie de tortuga gigante del género Chelonoidis Fitzinger, del Miembro inferior de la Formación Toropí/ Yupoí (Pleistoceno tardío/Lujanense), Bella Vista, Corrientes, Argentina. Ameghiniana, 50:287-297.

Zangerl, R, 1969. The turtle shell. In: C. Gans (ed.) Biology of the Reptilia, Academic Press, p. 311-339.

Zavala, C. \& Navarro, E. 1993. Depósitos fluviales en la Formación Monte Hermoso (Plioceno inferior-medio). In: CONGRESO GEOLÓGICO ARGENTINO, 12 Y CONGRESO DE EXPLORACIÓN DE HIDROCARBUROS PROVINCIA DE BUENOS AIRES, 2, 1993. Actas, Buenos Aires, p. 236-244.

Received in January, 2014; accepted in September, 2014. 
Apéndice 1. Especímenes utilizados a fines comparativos.

Appendix 1. Specimens examined for comparative study.

Chelonoidis chilensis: MHNSR 1600, 1601, 1602, 1603, 1604, 1605, 1606, 1607, 1608, 1609, 1610, 1611, 1612; dos ejemplares particulares sin número de colección.

Chelonoidis carbonaria: PC 3193, 4557, 6884, 37500, PC s/nº.

Chelonoidis denticulata: PC 284, 287.

Chelonoidis gringorum: AMNH 3366, MLP-75-II-5-2, MACN-CH-1009 y MPEF-PV-1049.

Chelonoidis nigra: $\mathrm{PC}$ sin número de colección.

?Chelonoidis australis: MLP-26-400 (Holotipo) MACN 5195.

?Chelonoidis gallardoi: MACN 5206 (Holotipo).

Chelonoidis lutzae: CTES- PZ 7391 (Holotipo).

Chelonoidis sp. de la Quebrada Saguayo, Bolivia: YPFB PAL 0932.

cf. Chelonoidis de la Formación Toropí/ Yupoí, Bella Vista, Corrientes, Argentina (Noriega et al., 2000): CTES- PZ 7101.

Trachemys dorbigni: MHNSR s/nº, UNNEC 11235. 